\title{
The CASE Histogram: Privacy-Aware Processing of Trajectory Data Using Aggregates
}

\author{
Maryam Fanaeepour • Lars Kulik . \\ Egemen Tanin - Benjamin I. P. Rubinstein
}

Received: date / Accepted: date

\begin{abstract}
Due to the high uptake of location-based services (LBSs), large spatio-temporal datasets of moving objects' trajectories are being created every day. An important task in spatial data analytics is to service range queries by returning trajectory counts within a queried region. The question of how to keep an individual user's data private whilst enabling spatial data analytics by third parties has become an urgent research direction. Indeed, it is increasingly becoming a concern for users. To preserve privacy we discard individual trajectories and aggregate counts over a spatial and temporal partition. However the privacy gained comes at a cost to utility: trajectories passing through multiple cells and re-entering a query region, lead to inaccurate query responses. This is known as the distinct counting problem. We propose the Connection Aware Spatial Euler (CASE) histogram to address this long-standing problem. The CASE histogram maintains the connectivity of a moving object path, but does not require the ID of an object to distinguish multiple entries into an arbitrary query region. Our approach is to process trajectories offline into aggregate counts which are sent to third parties, rather than the original trajectories. We also explore modifications of our aggregate counting approach that preserve differential privacy. Theoretically and experimentally we demonstrate that our method provides a high level of accuracy compared to the best known methods for the distinct counting problem, whilst preserving privacy. We conduct our experiments on both synthetic and real datasets over two competitive Euler histogram-based methods presented in the literature. Our methods enjoy improvements to accuracy from $10 \%$ up to $70 \%$ depending on trip data and query region size, with the greatest increase seen on the Microsoft T-Drive real dataset, representing a more than tripling of accuracy.
\end{abstract}

Maryam Fanaeepour $(\varangle) \cdot$ Lars Kulik $\cdot$ Egemen Tanin Department of Computing and Information Systems, University of Melbourne, Parkville, VIC 3010, Australia National ICT Australia (NICTA)

E-mail: $\{$ mfanaeepour,lkulik,etanin $\} @$ unimelb.edu.au

Benjamin I. P. Rubinstein

Department of Computing and Information Systems,

University of Melbourne, Parkville, VIC 3010, Australia

E-mail: brubinstein@unimelb.edu.au 
Keywords Aggregate Data - Count Information - Differential Privacy · Distinct Counting Problem · Euler Histograms · Location Privacy · Spatial Databases · Spatial Data Analytics

\section{Introduction}

The proliferation of location-based services (LBSs) has led to an ever-increasing amount of spatio-temporal data, and a growing need to process and analyse this data. A wide range of applications benefit from spatio-temporal data such as: finding a service in the vicinity of a user's location; determining the best site to set up a new facility such as a café, i.e., facility location planning; and traffic planning by monitoring the number of vehicles in a specific region [7]. However, significant concerns regarding an individual's data privacy can limit adoption [11]. Users naturally prefer privacy even when they grant third parties access to their data. Recent studies demonstrate that with growing usage of LBSs, individuals are becoming increasingly concerned about their privacy and shared information [26].

Even with only GPS trajectories, it is possible to track and identify a person via a reverse look-up inference attack [21]. In particular, many trips start or end at a user's persistent location e.g., home or work. Therefore, a trajectory ID is nearly as revealing as a person's ID.

Aggregation is a promising solution for privacy-aware trajectory analytics, as each individual user's trajectory need not be retained. There are two privacy models to address aggregate queries: the interactive and non-interactive settings [14]. In the non-interactive model, the database is sanitized and then released, to be used arbitrarily by any parties without limitation. In contrast, in the interactive setting, queries are submitted by users to the data provider, which acts as an intermediary between users and database. In order to protect the users' privacy, queries and/or the responses may be modified by the curator [13]. As discussed in [27], 'Non-interactive data release has been pursued aggressively in recent years because it avoids the costs and delays of implementing manual privacy safeguards'. As mentioned in the literature [9], 'histograms are natural candidates for privacy-preserving data sanitization'. The main contribution of this paper is a pair of non-interactive algorithms that compute sanitised histograms offline, and release these to a third party for answering subsequent range queries.

Our approach provides a qualitatively similar privacy guarantee to $k$-anonymity [38]. We introduce and bound the confusion level provided by our method in Section 6, formalising the idea that the trajectories consistent with our histograms should be numerous and indistinguishable. Moreover, we discuss attacker knowledge, as well as possible attacks and how to mitigate their success. We argue that the level of aggregation needs to be sufficient to provide a high level of privacy.

Fortunately a number of critical application scenarios require only aggregated trajectory data as a sufficient statistic. For example, aggregates are sufficient to answer the most common queries in traffic monitoring, i.e., "the number of cars passing through a specific query region during a specific time". In this paper, we focus on count information, i.e., the number of trajectories. Counting is one instance of several aggregate functions that are applicable to spatio-temporal data $[40,41]$.

Figure 1 depicts a typical data sharing scenario for privacy-aware data analytics, that is typical in applications such as quantified-self fitness apps, traffic monitoring, etc. Here three parties are involved: the intermediary party, which acts as service/data provider that collects the data, the user that generates the data, and the third party that requires a view on the user data to perform data analytics. Our core goal is to develop algorithms that supply 
aggregate statistics from historical location data to third parties while keeping user's data private. Our approach is for the service/data provider to release historical count histograms to third parties, who may then run range queries on this sanitized aggregated data.

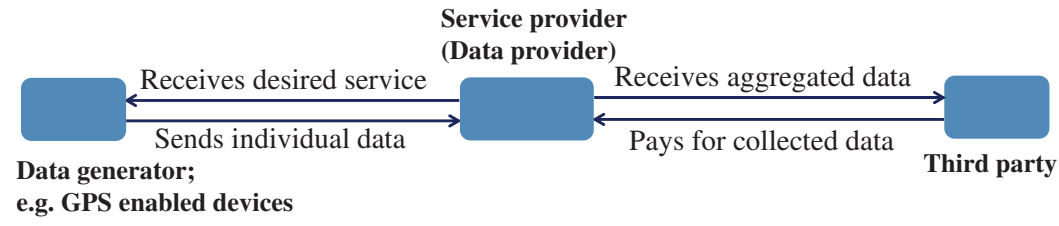

Fig. 1 A typical scenario for privacy-aware data analytics, where count information of privacy-sensitive data generators is collected by a service provider; the service provider then releases privacy-preserving aggregate counts to third parties.

An example that illustrates this scenario is mobile network operators, their customers and a business looking to open up a new retail space. Owing to the developments in technology and its ease of access, the increasing number of smartphone users can act as potential real-time data generators. The mobile network operator, acting as the service provider, could collect smartphone data and provide gathered statistics to the third party business. The most attractive locations from the business's perspective would be those places that are visited by considerable number of users. The core question of this paper is to find a solution to satisfy the needs of all parties: ensuring data quality for third parties while keeping users' data private.

\subsection{The Distinct Counting Problem}

A well-established spatial aggregate query is the distinct counting problem [7, 17,24,29], which is also known as the double counting problem. In this problem, space is partitioned into a set of pairwise-disjoint cells with each cell maintaining a count histogram data structure that counts objects intersecting with the corresponding cell. Given a query region, the goal of the distinct counting problem is to respond with a count of the distinct objects intersecting the query region. The challenge arises when objects exiting and re-entering the query region multiple times form a disconnected intersection with the query region. In such situations, cell-based counts may multiple count the number of distinct objects. The difficulty in maintaining counts for such objects, is that linking object segments is impossible without storing an ID per object. This challenge motivates us to propose a new technique that addresses this problem by introducing a notion of virtual counts. This challenge is of particular importance as there are many significant applications of aggregate trajectory data where privacy is a limiting concern.

Example 1 Figure 2 shows a moving object trajectory (e.g., a mobile phone user or a GPSenabled car) that exemplifies the challenge of the distinct counting problem. This object traverses 9 grid cells-with solid lines representing cell boundaries on the map. Circular points on the boundaries of grid cells demonstrate that the connectivity of the object path will be lost as it enters a neighbouring grid cell. As each cell independently maintains its own count, each segment contributes a count increment as shown by the highlighted "1"s. 


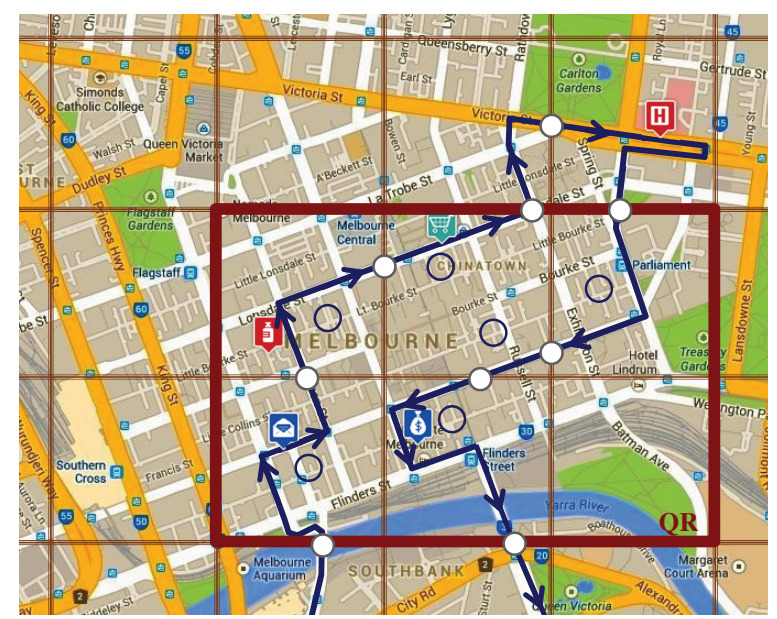

Fig. 2 An example of the distinct counting problem caused by a moving object trajectory visiting points of interest in the central business district of Melbourne, Australia. Each circled one indicates an increment to the corresponding cell count.

The query rectangle $(Q R)$, which is shown as a bold boundary, is covering 6 grid cells. As shown, the number of moving objects inside the query rectangle is erroneously computed as 6 instead of 1 since cell-based counts have no way to distinguish objects or share information (e.g., via an ID). The distinct counting problem is further compounded by the aggregation of many moving objects' trajectories common in real-world scenarios.

Where simple histograms suffer from double counting at the cell-count level, the Euler characteristic from the mid-1700s [42] serves as the basis for a series of more sophisticated data structures that eliminate this problem [3,37,47]. The general intuition is one of the inclusion-exclusion principle whereby the double counting of an object falling on two adjoining cells is corrected by an edge-crossing count; edge-crossing counts can also be corrected via vertex counts, and so on (for dimensions higher than 2). These techniques are usually designed for planar representations or entities such as polygons. The state-of-theart today, is the Distributed Euler Histogram (DEH) which counts trajectories of moving objects [47]. DEH correctly answers a simple question: how many distinct entries do trajectories make into a query region? However, DEH can still suffer from distinct counting in response to queries. In particular, the scenario in Figure 2 poses a challenge for DEH which calculates an incorrect answer of 2 (this holds for all other existing approaches; see Section 3.2 for more details on DEH). Next, we define these two related problems, studied extensively in the literature.

Definition 1 Given a partition of the plane, a set of objects, and a query region, the distinctentry counting problem is to compute the number of distinct connected components (or segments), of the objects intersected with the region. Distinct entry responses may only depend on aggregate cell-based counts.

In Figure 2, the number of distinct segments in the displayed query region is 2, while there is only one object. The difficulty in counting distinct planar objects can arise when the objects are not convex or are not completely covered by the query region. In this paper we aim to solve the challenging task of counting distinct objects which is often referred to 
as the "distinct counting problem". In particular we focus on counting trajectories and on rectangular partitions and query regions.

Definition 2 Given a partition of the plane, a set of objects, and a query region, the distinctobject counting problem is to compute the number of distinct objects intersecting with the query region. Distinct object responses may only depend on aggregate cell-based counts.

In real-world examples, trajectories are not limited to simple lines but can be selfintersecting paths, loops that return to their origin, or paths containing multiple cycles. A variety of trip patterns are possible, with several provided in an experimental study in Section 8.2. Consequently it is of the utmost importance to address the general distinct-object counting problem.

\subsection{Contributions}

In this paper, we discuss a privacy-aware trajectory data processing method using count information. We propose Connection Aware Spatial Euler Histograms (CASE Histograms) to solve the distinct-object counting problem. In CASE histograms, we preserve connectivity of a trajectory across cells without sharing any individual information between cell counts. The key insight is to planarize trajectories and then convert concave objects to being convex through the use of virtual counts. Our proposed method achieves a high level of accuracy, often-times releasing exact responses.

The main contributions of this work are as follows:

- We address the distinct-object counting problem by maintaining the connectivity of a trajectory without storing individual trajectory data. We propose the CASE histogram algorithm that counts the trajectories in query regions by applying a virtual count technique;

- The CASE algorithm preserves individual privacy through tunable aggregation. We provide the first theoretical analysis of privacy for Euler histogram-based methods including CASE;

- When users pre-process their trajectories into histogram deltas prior to submitting to the service provider, our approach achieves pan privacy — full access to the inner-state of our algorithm reveals no additional private information;

- Our approach provides third parties with highly accurate aggregated data, where no noise gets added to counts;

- We consider how differential privacy can be combined with Euler histograms in general, to further strengthen privacy against powerful attackers hoping to breach privacy by exploiting accurate responses to carefully crafted query regions; and

- We evaluate our techniques through extensive experimental studies on Melbourne, Manhattan and Beijing road networks as real-world examples. In addition, we test our technique on a real dataset, T-Drive. Our technique achieves a high level of accuracy and significantly outperforms the state-of-the-art.

The rest of the paper is organized as follows: the related work is reviewed in Section 2. In Section 3, we discuss background on Euler histograms for counting planar objects and an extension for counting trajectories of moving objects. Next, in Section 4, we develop our approach to solve the distinct-object counting problem. In Sections 5 and 6, we analyse the accuracy and privacy of CASE; and we develop variants of CASE that are alternatively more accurate and equally private $\left(\mathrm{CASE}^{+}\right.$algorithm) or less accurate but preserve differential 
privacy. In Section 7, we discuss practical guidelines for selecting the partition grid cell size. We analyse and compare our approach with the best competitive techniques in Section 8. Finally, Section 9 concludes the paper.

\section{Related Work}

The demand for location-based services (LBSs) has been increasing due to the advances in spatial-aware technologies such as GPS, RFID and GSM networks [11,16, 20,49, 19, 50, 17]. There exists a variety of applications that require spatial and spatio-temporal data analytics $[6,34]$.

Chow \& Mokbel [10] discuss the state-of-the-art in privacy-preserving techniques for spatial trajectory data, where applications require access to individual data. By contrast, several applications such as facility location planning [45] and traffic monitoring [7,31,39,41] do not need to work with individual data. In fact, in order to meet the third-party requirement and the user's privacy concerns, aggregation techniques $[8,33]$ as a privacy-aware method could be applied $[7,39]$. In these types of examples, moving objects' trajectories need to be pre-processed. Privacy-aware trajectory data processing using aggregates is the focus of this paper. Both storage space and privacy requirements motivate aggregation methods [7].

Xie et al. [46] propose a privacy-preserving system for traffic monitoring that aggregates vehicle IDs into partial IDs. Their idea is based on $k$-anonymity [38] in which every record released shares identifying information with at least $k-1$ other individuals. Higher levels of anonymity incur degraded accuracy in a trade-off controlled by the number of trajectories sharing a partial ID. Although partial IDs may not always breach privacy, requirements of higher accuracies lead to partial IDs that do effectively act as pseudo IDs. Our work does not require storage of IDs, and offers two settings: in one, users transmit trajectories to a trusted service provider who only retains (and releases) aggregated count data; in the other, users may transmit count increments only. This second setting constitutes a form of privacy known as pan privacy [15] in which even a full audit of the service provider would not uncover additional privacy-sensitive data.

We argue that map-matching attacks [44] are mitigated by count-based approaches. Indeed each cell in our approach acts like a mix zone [4,5] (a region in which location data is not transmitted), since counts of many trajectories crossing cells are combined without identification. Section 6 discusses the relationship between level of aggregation and our notion of 'confusion level' - the number of indistinguishable trajectories consistent with our histograms. This guarantee is very similar to $k$-anonymity, which is a leading privacy framework popular due to its usable balance of privacy and utility. We discuss in Section 6.1 and 6.4 that cell size and the duration of data gathering must be sufficiently large to guarantee large confusion levels (i.e., large $k$ in $k$-anonymity). Differential privacy is a strong notion of privacy that guarantees released statistics do not depend significantly on any individual datum - an attacker with access to almost all other data cannot use released statistics to determine an unknown individual's datum [14]. Our approaches are complementary to differential privacy. Without any aggregation, randomised query responses that preserve differential privacy suffer higher degradation to accuracy, while differential privacy can add a stronger level of privacy guarantee on top of aggregation. We propose for the first time the (optional) combination of differential privacy with Euler histogram methods in general, in Section 6.5.

While map-matching attacks [44] are mitigated by count-based approaches, these techniques suffer a common challenge: the distinct counting problem (known as the double 
counting problem $)[24,43,29,30,25]$. This problem occurs when a spatial or spatio-temporal object, e.g., trajectory, remains in a query region for several time stamps in the query interval and is counted multiple times. Traffic monitoring, for example, could be seriously impacted by inaccuracies due to multiple counting.

The distinct counting problem has been discussed in the literature for spatial data since the late 1990s [3,32]. Papadias et al. [31] propose the aRB-tree (aggregate RB-tree) to index spatial data, where the count of cars related to the area of a tree node is stored. Queries are answered by summing up these counts while traversing the data structure. However, the approach still suffers from the distinct counting problem.

Tao et al. [39] identify this problem as coming from the use of the aRB-tree, and they discuss the difficulty with providing accurate answers for distinct counting queries. They opt not to focus on exact counts and propose using sketches as an approximation method to address this problem. This approach is based on probabilistic counting which is approximate. Our goal in this paper is to develop approaches that can produce accurate aggregated responses ( $c f$. Sections 5 and 8.4) for certain queries ( $c f$. Section 3, Remark 2).

Yaagoub et al. [51] propose an approach similar to the aRB-tree approach of Papadias et al. [31]. As well as using a Quadtree-based index, their approach considers materialized views for the internal nodes in contrast to [31]. However, unlike the work in this paper the object ID is retained, which posses a threat to privacy.

Loo [23] explores buffer zones in road network databases, to define standardized regions that encompass roads and highways for the purposes of linking road network data with vehicle accident reports. While their work is not motivated by privacy, the use of a buffer zone causes problems of double counting. In particular, for those areas that have narrow and dense roads, and are near junctions. In this setting, the geometry of the object is the primary cause of double counting.

All of the aforementioned works differ fundamentally from our aim: to answer coarse range queries accurately while preserving the privacy of users. Our approach is to maintain the connectedness of trajectories or objects that are disconnected by cell boundaries.

Our work builds on the Euler histogram which was first introduced by Beigel \& Tanin [3] to address the distinct counting problem for rectangular objects, using the Euler characteristic [42]. We describe Euler histograms in Section 3. Sun et al. [36,37] further develop histograms as an approach to answering range queries, and contribute lower bounds on storage and efficient approximation algorithms for a range of query types. Distributed Euler Histograms (DEHs) have been proposed more recently to address aggregate queries on moving object data in distributed settings [47]; the distinct entries problem is proposed and tackled rather than distinct-object counting problem. Recently, Leonardi et al. [22] consider a countbased approach and demonstrate accuracy results that can outperform others. Their approach is similar to DEH [47], as they also consider crossing and overlapping of trajectories with the edges and faces of grid cells to compute the number of distinct objects. The experimental comparison [22] between their Euler histogram-like approach and sketches (probabilistic counting) [39] shows that count-based histograms outperforms the randomised technique. Our approach can outperform DEH and consequently other existing techniques, as is demonstrated experimentally in Section 8. We have in parallel explored combining the approach developed first in this present paper with tree data structures to reduce the storage requirements of histograms in [48]. 


\section{Background}

In this section, we review the Euler histogram as a technique to count convex planar objects [3] and its extension DEH for moving objects' trajectories [47]. We offer a summary and new insight into the limitations of these existing approaches to distinct counting.

As we restrict our attention to rectangular partitions and query regions, without loss of generality, we can fully represent a range query in terms of its minimum and maximum row and column numbers. These choices are discussed in Remark 2, including extensions to other geometries (See also Section 7 for further analysis regarding the grid cell size and query rectangle shapes).

\subsection{Counting Planar Bodies: Euler Histograms}

Beigel \& Tanin [3] extended Euler's characteristic to count the number of objects in a cellpartitioned space. The Euler histogram is composed of three types of counts as follows:

- Face counts represent object counts associated with each cell. A cell's face count is equal to the number of objects intersecting with that cell;

- Edge counts represent object counts associated with each edge between two neighbouring cells. An edge's edge count is equal to the number of objects crossing that edge; and

- Vertex counts are the number of objects intersecting with a vertex incident to four cells (and four edges).

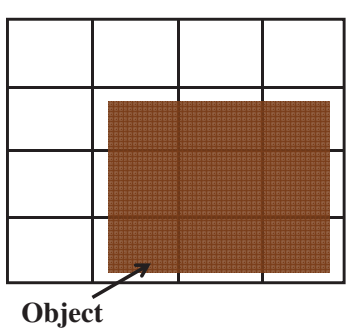

(a)

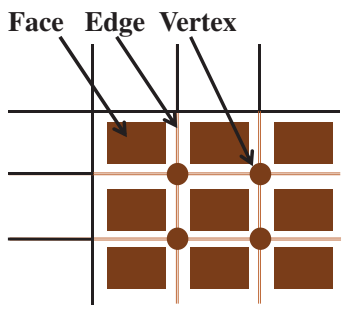

(b)

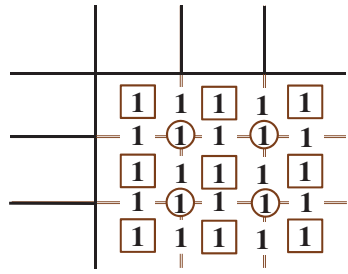

(c)

Fig. 3 An example (a) partition with one object from Example 2; (b) the faces, edges and vertices overlapping with the object; and (c) the resulting counts in the Euler histogram.

Example 2 Figure 3 demonstrates the concept of Euler histogram with a $4 \times 4$ partition and one rectangular object occupying the bottom-right $3 \times 3$ cells. Rather than a single count per cell, as in a simple histogram, the Euler histogram maintains counts for all faces, edges and vertices. The faces, edges and vertices impacted by the object are highlighted in Figure $3 b$ and the resulting counts are displayed in Figure 3c. In total 9 cells have face count 1, 12 edges have count 1 (6 horizontal edges and 6 vertical edges), and 4 vertices have count 1 . All other counts in the histogram are zero. 
Given a rectangular query region that is a union of cells, we respond to the query with the number of objects in the region, by combining up relevant face, edge and vertex counts:

- Total face count $F$ is the sum of face counts of those cells contained in the query region;

- Total edge count $E$ is the sum of edge counts of those edges whose two incident cells are contained in the query region; and

- Total vertex count $V$ is the sum of the vertex counts of those vertices whose four incident cells are contained in the query region.

With these three counts in hand, the object count is calculated for response, as:

$$
N=F-E+V .
$$

This is a form of the inclusion-exclusion principle, whereby an overall count has a firstorder estimate given by the sum of face counts. However objects that overlap multiple cells are thus multiple counted. Edge counts bound the degree of this double counting, however correcting by subtracting edge counts is still not exact: $F-E$ also needs to be corrected by vertex counts as objects that overlap intersecting edges are multiple counted by the edge count $E$. This is very similar to correcting the union bound in probability theory with probabilities of event intersections. In higher dimensions the expression involves an alternating series of corrections [3].

Example 3 Returning to Example 2, consider now a query region equal to the entire space. Applying the rules of combining face, edge and vertex counts yields $F=9, E=12, V=$ 4. Equation (1) then leads to the correct object count of $N=F-E+V=9-12+4=$ 1. However, a simple histogram, that would only consider the face counts, would give an erroneous count of 9 .

\subsubsection{Accuracy of Euler Histograms}

In many circumstances Euler histograms produce accurate counts. However they are known to multiple count in certain settings.

Remark 1 A sufficient condition for correctness of Equation (1), is that all objects are convex planar bodies. We demonstrate in the following example that convexity is not necessary however. In general, objects being disconnected leads to inaccurate counts. Note that connected objects can become disconnected e.g., a concave object not contained by a query region (cf. Example 4).

Example 4 Figure 4 shows a concave object and a query rectangle $(Q R)$ intersecting only part of the object. As a result, within the region the object is disconnected (cf. Figure 4a). Note that by the rules of combining vertex and edge counts, there are no such counts inside the query rectangle and $F=2$ (cf. Figure $4 c$ ) yielding an erroneous count of $N=F-E+$ $V=2-0+0=2$. If the query region were expanded to contain the concave object, it is easy to see that $F=8, E=9, V=2$ yielding $N=1$. This proves that convexity is not necessary for correctness.

It is clear that trajectories behave very differently to convex bodies in general, and so could lead to inaccuracies without further modification to the Euler histogram data structure. We explore trajectories in the next section. 


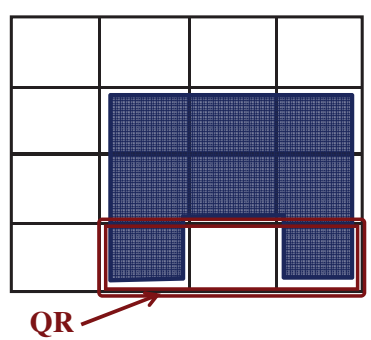

(a)

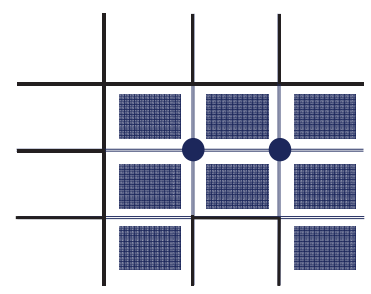

(b)

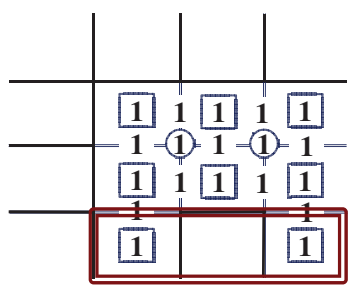

(c)

Fig. 4 Euler histograms from Example 4 with: (a) a concave object not contained by the query region (QR) becomes disconnected; (b) the impacted faces, edges and vertices; and (c) the corresponding counts.

Remark 2 In this paper we have chosen to focus on 1. rectangular partitions (having a convex partition is sufficient), 2. convex query regions (rectangles) that are unions of cells, and 3. to sum only those counts that are "fully covered". Regarding Assumption 2, query region convexity is a necessary condition for accurate Euler histogram counting of convex planar bodies. While we can service arbitrary convex query regions with minor modifications to the count-summing rules (Assumption 3): if a cell is contained in the query region or partially overlaps it, then its counts contribute to the sums. Therefore the effective query region is in fact a union of cells overlapping the given convex query region. Moreover the rectangular cell partition, Assumption 1, can also be relaxed to any convex partition. Indeed previous work applying Euler histograms [47] have considered Voronoi partitions induced by centroids at sensor locations, and query rectangles that are unlikely to be unions of such cells. However, like the case of non-rectangular convex query regions, such partitions are unlikely to overlap completely with given query regions. None of the results in this paper are limited to the regular partitions and regions discussed. However, when the assumptions are relaxed, responses to a given query region do not only count objects falling within the query region, but rather count objects in cells that overlap the query region-the effective query region is still a union of cells. Our treatment avoids the need to consider effective query regions as distinct from given query regions, thus simplifying our exposition while restricting the source of errors to be only from multiple counting.

\subsection{Counting Trajectories: Distributed Euler Histograms}

The principles of counting planar objects can also be applied to moving points represented as trajectories, which we discuss in this section. Distributed Euler Histograms (DEHs) [47] service aggregate queries on moving object data in distributed settings. This approach applies Equation (1) with one main modification: there are no vertex counts. DEH supports distributed query responses by having each cell store its own associated face and edge counts.

In DEH, each cell's face and edge counts are incremented every time a trajectory enters a cell: a trajectory that passes through a cell and then re-enters once more through the same edge, contributes 2 to both face and edge counts. The rules for summing face and edge counts for a given query region produces $F$ and $E$ in the same way as for Euler histograms. No vertex counts are maintained in DEH however, since a trajectory is merely a poly-line that "does not cover" any vertex [47]. 


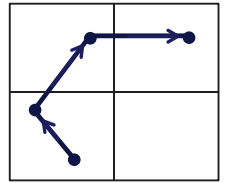

(a)

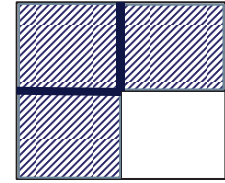

(b)

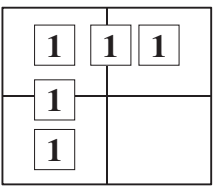

(c)

Fig. 5 A (a) moving object's trajectory; (b) an equivalent concave body corresponding to cells covered; and (c) the histogram counts for both objects.

Example 5 Figure 5 a presents the Euler histogram for a moving object's trajectory on a $2 \times$ 2 grid. Due to the rules of incrementing counts, the trajectory shown and the concave planar body in Figure $5 b$ are indistinguishable: in both cases the histogram counts of Figure $5 c$ result.

The following expression represents the response of DEH for a given query region:

$$
N=F-E \text {. }
$$

\subsubsection{Accuracy of Distributed Euler Histograms}

The intuition behind Equation (2) is that whenever a trajectory enters a grid cell, we increase one count for that the cell's face, and when it leaves we decrement the related edge count. As each trajectory ultimately finishes in a grid cell, then only the last visited grid cell will have an edge-uncorrected face count of one. However, depending on the shape of the trajectory and the query rectangle, this expression cannot always provide an accurate response.

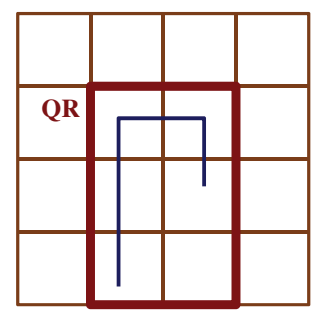

(a)

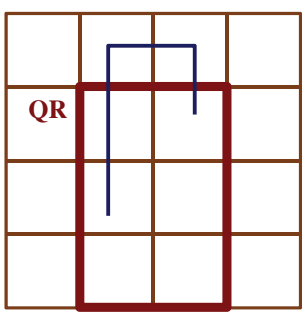

(b)

Fig. 6 An example that demonstrates (a) when DEH can correctly count trajectories; and (b) when the response is inaccurate.

Example 6 Figure 6 demonstrates the key limitation of DEH, when a trajectory re-enters a query region; i.e., the intersection of the trajectory with the query region is disconnected. In Figure $6 a$, there is one trajectory which is completely inside the query region, and therefore connected. Equation (2) computes a correct count of $N=F-E=5-4=1$. However, as depicted in Figure 6b, once only segments of the trajectory lie inside the $Q R, D E H$ no longer solves the distinct-object counting problem. Although there is only one object inside the $Q R$, the algorithm estimates $N=F-E=3-1=2$. 


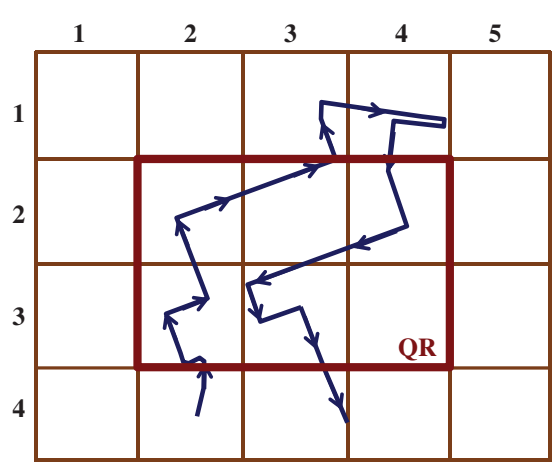

(a) A trajectory on a $4 \times 5$ grid

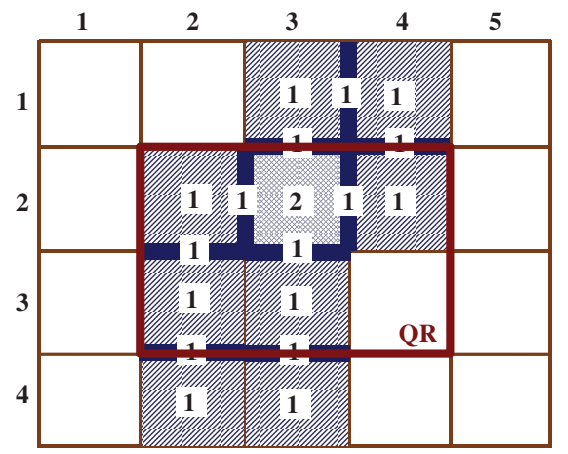

(b) Faces and edges covered

Fig. 7 A sample trajectory that causes the distinct counting problem and the application of Euler histograms that does not address it.

Example 7 Consider again Example 1 from Section 1.1, with a single object-a similar setting but more complex than the previous example. Figure 7 a repeats the pertinent details of the example, showing the partition, trajectory and query region only. Recall that under a simple histogram that considers only face counts, we get 6 as the reported number of objects in the query region. By applying Equation (2), with $F=6$ and $E=4$, we arrive at $N=F-E=6-4=2$, which is closer to the correct count, however still suffers from double counting. In fact, DEH addresses the distinct-entry counting problem rather than the distinct-object counting problem (Definitions 1 and 2). As in the limitation discussed above for Euler histograms, the problem for DEH here occurs as the query region does not fully cover the trajectory. This trajectory loses its connectivity within the query region (cf. Remark 1). Figure $7 b$ shows the faces and edges of the cells involved with this trajectory. As depicted in Figure $7 b$, cell $(2,3)$ is different from the others as the trajectory traverses this cell twice and it accordingly receives a count of two. As discussed in Example 5 and depicted in Figure 5, a simple trajectory corresponds to a (possibly concave) planar body. Since the rules of updating face and edge counts in DEH recounts a trajectory that re-enters a cell, such cases correspond to superposing multiple (possibly concave) bodies, one per segment within the query region. This is apparent in Figure $7 b$ where the affected cells shown in grey correspond to the union of the two segments' cells; the only overlapping cell being $(2,3)$.

\section{Algorithm: CASE Histograms}

We now propose the Connection Aware Spatial Euler (CASE) histogram to address the distinct-object counting problem. In CASE histograms, we preserve the connectivity of trajectories that may be disconnected by a query region, and in so doing achieve higher accuracy for the same level of privacy. CASE histograms leverage several key ideas:

- Planarization. In DEH, trajectories and the corresponding planar union of intersecting cells are indistinguishable from counts alone. In CASE, we make this connection explicit by planarizing trajectories;

- Processing entire trajectories. In DEH a trajectory is processed by segment, and so there can be multiple superposed planar bodies contributing to counts—any cells be- 
longing to the bodies of multiple trajectory segments will suffer double counting. CASE processes a trajectory in its entirety, and so only a single planarized body is produced;

- Convexity. To avoid having concave planarized bodies that become disconnected by pathological query regions, we grow the planarized bodies to their convex minimum bounding rectangle (MBR) [35]. Convexity is sufficient for Euler histogram correctness (cf. Remark 1); and

- Completing virtual counts. Finally, the expanded MBR containing a trajectory may include cells that do not intersect with the trajectory. These cell counts are not incorporated in the counts from the trajectory alone, and so we introduce virtual counts to complete them. Since the object being counted is a planar body, we include virtual vertex counts, but no real vertex counts for the non-planar trajectory.

Given an observed moving object's trajectory we carefully increment our CASE histograms. The following represents a sketch of the algorithm. Some additional steps are taken to improve accuracy and efficiency-the full details are given in Section 4.1 and in the accompanying Algorithms 1 and 2.

- Identify the MBR of the trajectory, in particular the min/max rows and columns specifying the rectangle;

- Consider the MBR as a planar object to be counted by the Euler histograms: any faces, edges, vertices that would be counted in the Euler histograms for the MBR, but have not been counted for the trajectory (previous step) should be counted by virtual face, edge and vertex counts; and

- Process the observed trajectory/object by incrementing each intersecting cell's face and edge counts following the rules of Euler histograms (not the segment-wise rules of $\mathrm{DEH})$.

To compute the response value, we incorporate the counts $F, E$ and virtual counts $F^{\prime}, E^{\prime}$ and $V^{\prime}$ as:

$$
N=\left(F+F^{\prime}\right)-\left(E+E^{\prime}\right)+V^{\prime} .
$$

It is apparent, that the virtual counts are completing face/edge/vertex crossings that are not counted in $F, E$.

Example 8 As an example consider Figure 8 a which revisits our running example of the Melbourne central business district. The figure shows the MBR and its representative min/max cells. Next, Figure $8 b$ depicts the cells of the planarized MBR that intersect with the trajectory (dark faces) and do not intersect (light virtual faces); also shown are the corresponding edges, virtual edges and virtual vertices. These are again highlighted in Figure $9 a$. Finally, Figure $9 b$ displays a query rectangle alongside the histogram counts. Note we have $F=5, F^{\prime}=1, E=4, E^{\prime}=3, V^{\prime}=2$. Then applying Equation (3), we arrive at the correct distinct object count of $N=\left(F+F^{\prime}\right)-\left(E+E^{\prime}\right)+V^{\prime}=(5+1)-(4+3)+2=6-7+2=1$.

Virtual lines ( $c f$. Figure 10) can be drawn horizontally/vertically throughout an MBR to determine which cells are impacted by the MBR and therefore are included in count calculations for any given query region. In future work, if we were to planarize trajectories to non-rectangular bodies, virtual lines would prove useful in computing convex hulls.

\subsection{Algorithm Details}

In this section, we discuss details of the CASE histogram approach ( $c f$. Algorithms 1 and 2). We use the following basic structures in our algorithms. 


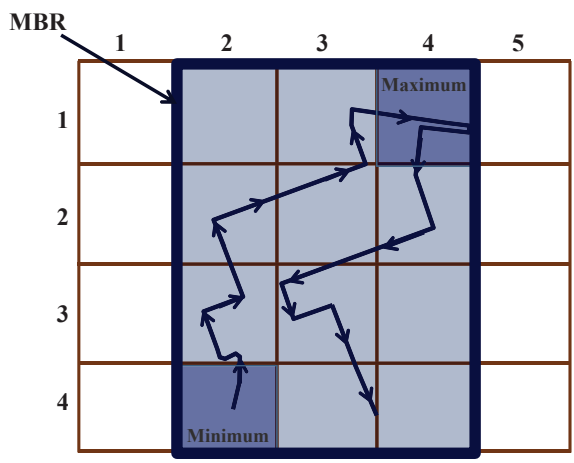

(a)

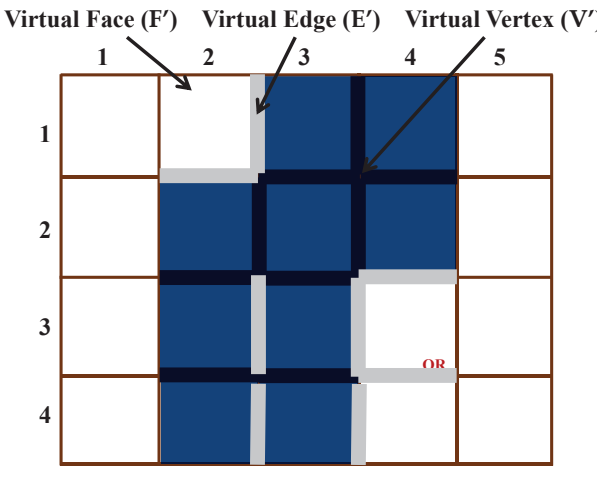

(b)

Fig. 8 (a) Extracting the minimum and maximum grid cells of a trajectory to compute its MBR and converting it to a convex planar object. (b) CASE histograms; Virtual Face, Virtual Edge and Virtual Vertex.

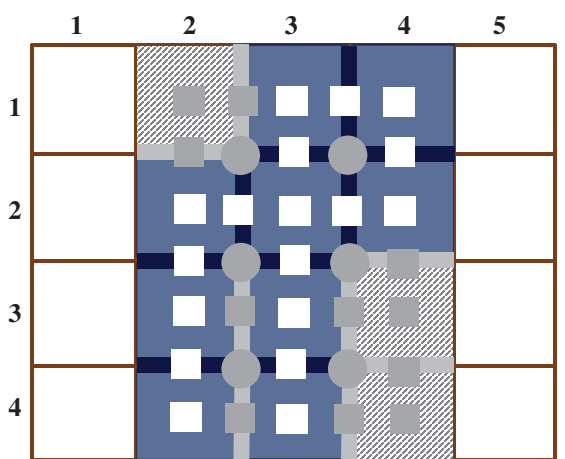

(a)

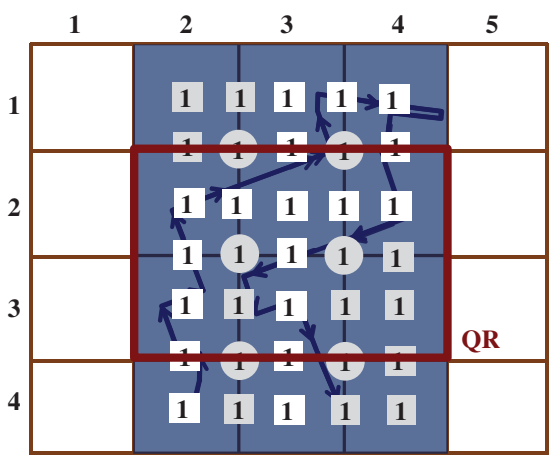

(b)

Fig. 9 (a) CASE histograms; and (b) virtual counts, for Figure 8.

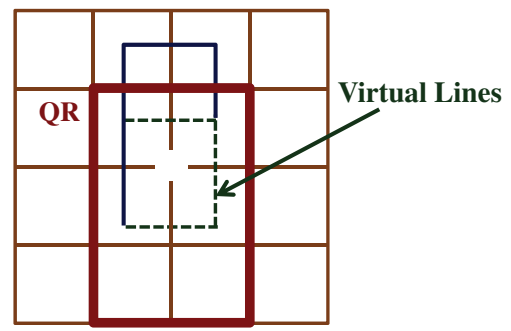

Fig. 10 "Virtual lines" (the dashed lines) that preserve the connectedness of an object.

- Trajectory $\mathscr{T}$ : A full trajectory of a moving object is a sequence of $n$ visited nodes $\mathscr{T}=\left(n_{1}, \ldots, n_{n}\right)$, for some $n$

- Link $\ell_{j}$ : Every two consecutive nodes, $n_{j}$ and $n_{j+1}$, of a trajectory induces a link composed of two lists: $\ell_{j}=\left(\left(c_{1}, \ldots, c_{s}\right),\left(e_{1}, \ldots, e_{s-1}\right)\right)$ : 
- Cell ID $c_{k}$ : The ID of a grid cell corresponds to the row and column index of the cell, e.g., $c_{k}=(2,3)$ means row 2 , column 3 . A link contains all IDs of cells intersecting with that link; and

- Edge ID $e_{k}$ : The ID of a cell's boundary edge corresponds to the pair of incident cell IDs. A link contains the IDs of all cell edges crossed by that link.

Both construction and query response algorithms deviate from the above design, in ways that can boost efficiency and accuracy. For example, during construction, we only concern ourselves with virtual counts if it is determined that the trajectory will indeed cause double counting problem without them.

Stage 1: Cell and Edge Retrieval. In CASE histogram construction (Algorithm 1), we take every trajectory path as a list of node IDs (input to the algorithm) and retrieve the related cell IDs and cell boundary edge IDs. If the current extracted cell ID exists in the cell ID list, it will not be added to the list to avoid repetition. This happens if two consecutive links are located in the same cell or the trajectory re-enters a cell. In both cases, the related cell ID should be considered only once. Furthermore, two consecutive links could cross the same edge of a cell. Here, we need to consider only one edge ID in the extracted edge ID list. In the case of a vertex crossing, we slightly perturb the trajectory so that the link crosses to one side of the vertex. This perturbation to the retrieval process can lead to small off-by-one errors in counts, for certain QRs. However this case does not occur in any of our datasets or range of experimental partitions, and so we deem resulting errors to be negligible.

Stage 2: Detecting the Distinct Counting Problem. While we may assume we will always suffer from the distinct counting problem, it is more efficient to detect when this is the case and otherwise not expend resources maintaining virtual counts. For this, we check the cell IDs in the extracted cell ID list. If there is a cell ID that has the same row or column number with another cell ID, but they are not neighbours and no connection link exists in the edge ID list, then the distinct counting problem occurs.

Stage 3: Planarization. In this final stage, the poly-line trajectory will be converted to a "planar" object if the distinct counting problem has been detected. That means, in addition to adding more cell IDs and edge IDs as virtual faces and virtual edges, vertex IDs associated with the cell IDs will also be added to the list as virtual vertices. In a nutshell, we have a list of cell IDs and edge IDs that this trajectory is intersecting with. Moreover, virtual faces, virtual edges and virtual vertices need to be added if the distinct counting problem occurs. For all of the extracted and computed cell IDs, edge IDs and possibly virtual counts, we will add one count to their related face, edge histograms, virtual face, virtual edge and virtual vertex histograms [lines $27-37$ ]. If the distinct counting has not occurred we do not need virtual counts [Stage 3 , lines $39-42]$.

In Algorithm 2, we elaborate on the range query procedure based on CASE histograms. The face count will be computed using the QR coordinates. If there is no real count inside the $\mathrm{QR}$, then we do not need to consider further count histograms, either real or virtual. The total number of objects will be zero, [lines 1-3]. Otherwise, the number of rows and columns corresponding to the requested $\mathrm{QR}$ will be checked. If the $\mathrm{QR}$ has only one row or one column, the sum of edge counts will be calculated as well as the total number of counts for virtual faces and edges [line 5-9]. Since there is no vertex constructing the QR, then there is no vertex count, so this parameter in Equation (3) will be zero. Thus, there are only real and virtual faces and edges counts to be considered. If there is any vertex, the sum of virtual vertices will be computed and Equation (3) will be applied [line $11-13$ ]. 


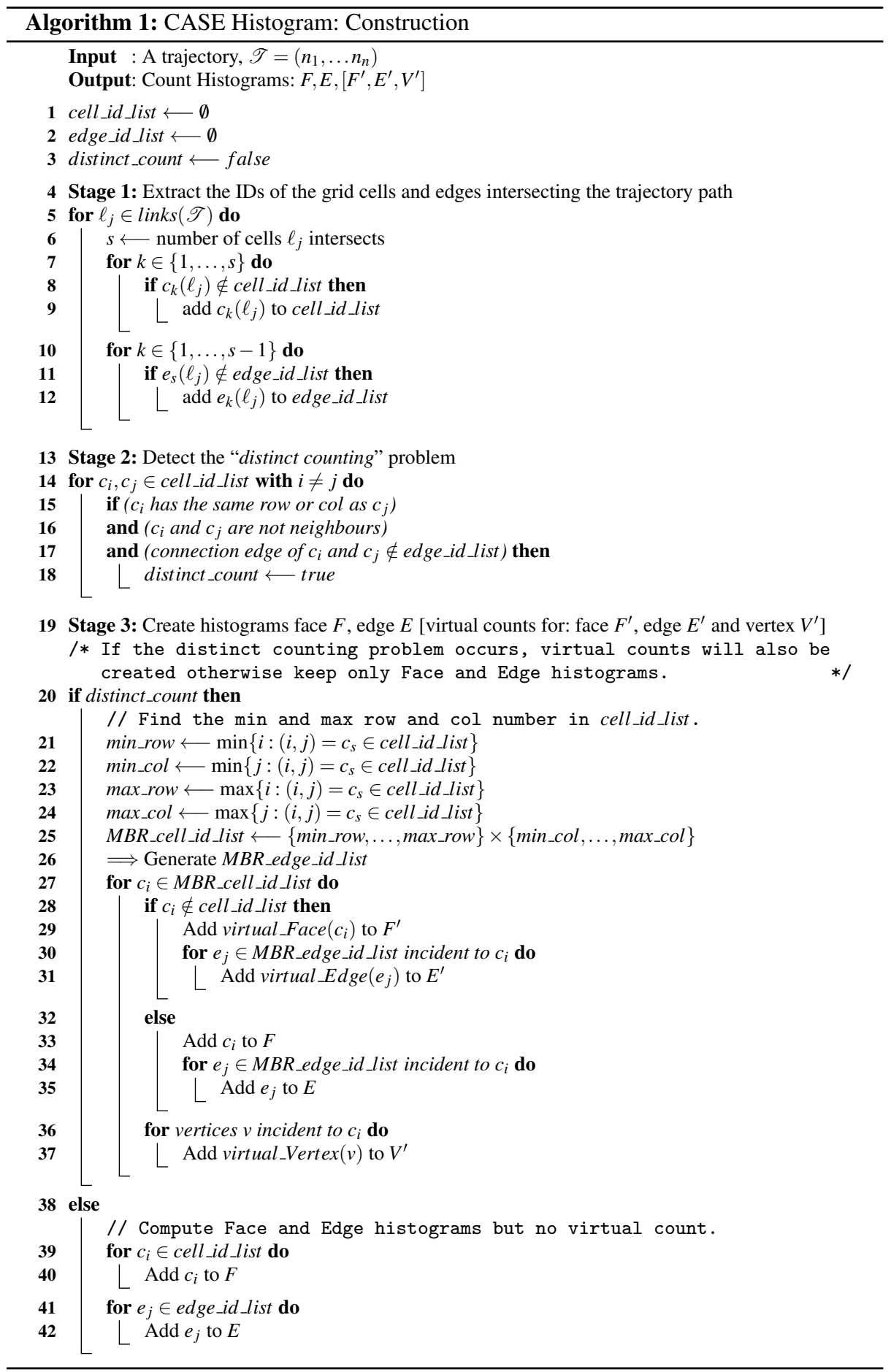




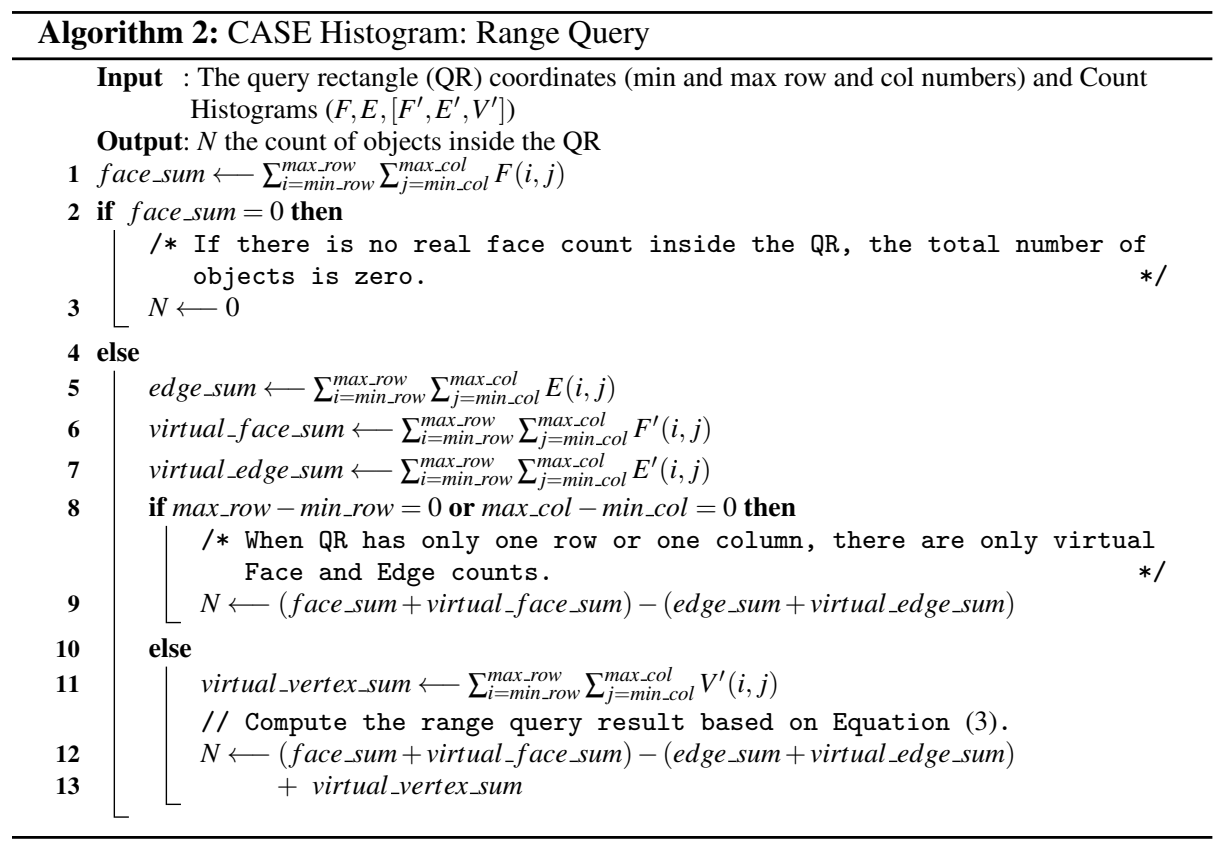

\subsection{Computational Complexity Analysis}

Regarding the query computation, the cost $O(n \times m)$ in the worst case corresponds to the number of grid cells covering the selected area, $n \times m$, in which $n$ is the number of rows in the partitioned map and $m$ is the number of columns. The number of grid cells does not change with respect to the input number of trajectories, it only changes when the grid cell size changes. In regard to the pre-computational process, the complexity $O(T \times n \times m)$ is also related to the number of trajectories $T$. In an offline procedure, the service provider executes a pre-computation once and sends it to a third party to run range queries over an extended period. In this way the cost of pre-computation is amortized. In general, the size of the dataset (number of trajectories), the length of a trajectory, and the number of grid cells are those factors that can affect the running time of this algorithm. However, the two latter factors will be constant and limited to the grid size and in the worst case if a trajectory passes all the grid cells would be $n \times m, n$ as the number of rows in the partitioned map and $m$ as the number of columns.

\section{Accuracy Analysis}

The principle behind the CASE histogram is to reduce trajectory counting to convex planar body counting: as discussed in Section 3.1 ( $c f$. Remark 1), convexity is sufficient for the accuracy of Euler histograms. On the other hand trajectories can be highly non convex, and become disconnected when restricted to a relatively small query region. The set of real and virtual counts, the rules for updating and summing them, culminating in Equation (3), form this reduction. As such, it is clear that where a query region does not only intersect virtual 
cells for an object, the CASE count is correct by the same argument as for Euler histograms. Virtual counts complete the bookkeeping of the planarized body.

As we have seen for the running Melbourne example, CASE accurately solves the distinct-object counting problem while state-of-the-art DEH solves only the simpler distinctentry counting problem ( $c f$. Example 8 ). We will supply additional experimental evidence in the sequel. However, the basic CASE algorithm can still suffer from inaccurate counts.

Example 9 Figure 11 demonstrates a case in which virtual counts may lead to an error for a smaller QR: all three trajectories T1,T2 and T3 in the figure can suffer from the distinct counting problem. Therefore, we convert them to a polygonal object via virtual counts. As discussed in Section 4.1, when the grid cells of a range query have only virtual counts, those counts are not considered. It is possible for smaller query rectangles to intersect with virtual counts of an object that is completely outside of the $Q R$. In this case, we have real counts inside the query rectangle in addition to the virtual counts. Therefore, we will get an extra count of the object. This is the detected source of error for CASE histograms. As shown, Trajectory $T 2$ is completely outside the QR but its related virtual counts are inside the $Q R$. The dotted lines inside the $Q R$ are the virtual lines corresponding to trajectory $T 2$. Since we have the full trajectory of $T 1$ and a part of $T 3$, we need to consider both real and virtual counts. Therefore, we will have one more object in the reported result: From grid cell $(3,4)$ to $(6,6)$, we can extract one virtual object to address the identified problem: we have 9 virtual faces, 17 virtual edges and 9 virtual vertices related to trajectory T2. Therefore, the number of virtual objects is $N=F^{\prime}-E^{\prime}+V^{\prime}=9-17+9=1$.

\begin{tabular}{|c|c|c|}
\hline \multicolumn{2}{|l|}{ 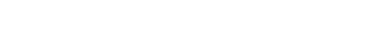 } & 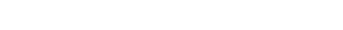 \\
\hline \multicolumn{2}{|l|}{ 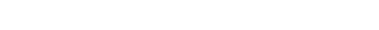 } & 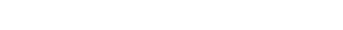 \\
\hline \multicolumn{2}{|l|}{ 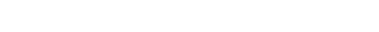 } & 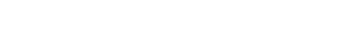 \\
\hline \multicolumn{2}{|l|}{ 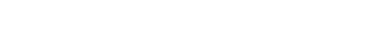 } & a \\
\hline \multicolumn{2}{|l|}{ 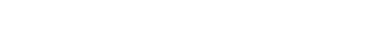 } & 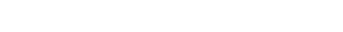 \\
\hline \multicolumn{3}{|c|}{ 国速雨 } \\
\hline \multicolumn{3}{|c|}{ 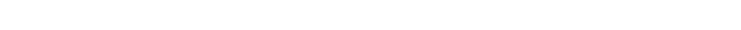 } \\
\hline \multicolumn{3}{|c|}{ 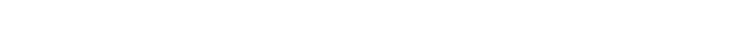 } \\
\hline \multirow{3}{*}{\multicolumn{3}{|c|}{ 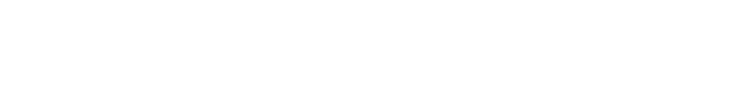 }} \\
\hline & & \\
\hline & & \\
\hline \multicolumn{3}{|c|}{ 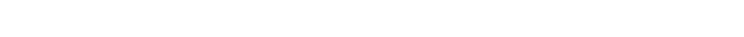 } \\
\hline \multicolumn{3}{|c|}{ 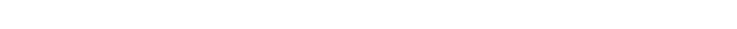 } \\
\hline \multicolumn{3}{|c|}{ 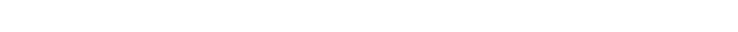 } \\
\hline \multicolumn{3}{|l|}{ 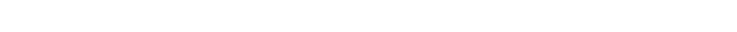 } \\
\hline \multicolumn{3}{|c|}{ 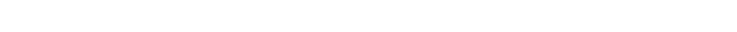 } \\
\hline \multicolumn{3}{|c|}{ 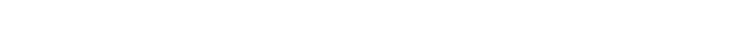 } \\
\hline \multicolumn{3}{|c|}{ 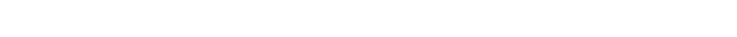 } \\
\hline \multicolumn{3}{|c|}{ 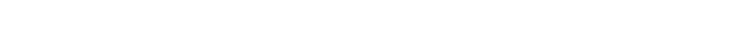 } \\
\hline \multicolumn{3}{|c|}{ 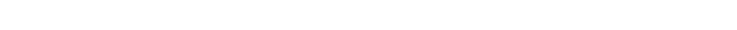 } \\
\hline \multicolumn{3}{|c|}{ 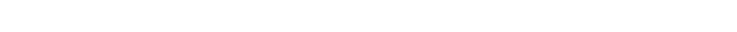 } \\
\hline \multicolumn{3}{|c|}{ 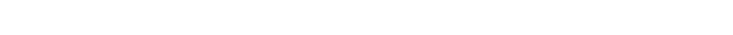 } \\
\hline
\end{tabular}

Fig. 11 Virtual counts for smaller QRs in CASE histograms can lead to double counting.

As this particular error occurs for specific trajectory shapes and the very small query regions, the CASE histogram overall provides a very high level of accuracy regardless of various parameter settings (see Section 8). We propose $\mathrm{CASE}^{+}$in the next section, designed 
to remove some unnecessary virtual objects. While we have provided one rule, further rules may be possible and can be developed in future research.

\subsection{Eliminating Unnecessary Virtual Counts: CASE ${ }^{+}$}

As shown in Figure 11, some virtual counts are not necessary, and lead to errors for smaller QRs. To address a special-case problem with virtual counts demonstrated in the previous section, we propose a rule to remove the virtual counts related to objects which are completely outside the QR and fully cover the QR.

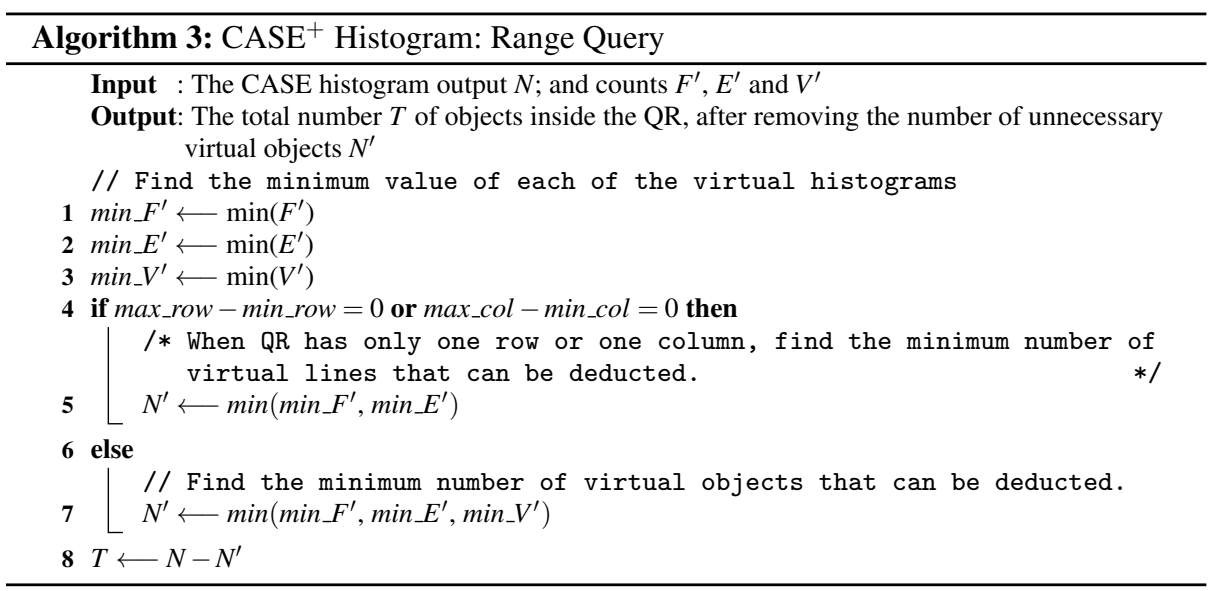

Given a Query Rectangle (QR) that is an $m \times n$ grid, the number of grid cells (faces) inside the QR equals to $m n$, the number of vertices is $(m-1)(n-1)$, and the number of edges $m(n-1)+n(m-1)$. As discussed earlier, CASE checks all $m n$ grid cells of the QR. If there are only virtual counts in the requested query rectangle, i.e., there is no real count, then the returned number of objects is 0 .

The above rule means that all the object(s) causing the distinct counting problem are outside the QR. Thus, we do not consider the virtual counts and report no object inside the QR. However, if inside the requested QR there are real counts as well as virtual counts, we need to compute the number of objects based on the CASE expression, Equation (3). In the $\mathrm{CASE}^{+}$algorithm ( $c f$. Algorithm 3), we check if we have at least 1 virtual count per grid cell and grid edge in the QR. If all the $(m-1)(n-1)$ vertices have at least 1 virtual count as well, then we compute the number of virtual object(s) that we can extract by deducting 1 from the $F^{\prime}, E^{\prime}$ and $V^{\prime}$ histograms per cell, edge and vertex, to produce $N^{\prime}$. This means that the number of virtual objects that we can remove from the total reported result is the minimum amount between the minimum of virtual histograms, $F^{\prime}, E^{\prime}$ and $V^{\prime}$ :

$$
N^{\prime}=\min \left(\min \left(F^{\prime}\right), \min \left(E^{\prime}\right), \min \left(V^{\prime}\right)\right)
$$

In other words, for each planar object superimposed by an $m \times n$ grid, we have the following:

- No. of vertices $V=(m-1)(n-1)$; 
- No. offaces (cells) $F=m n$; and

- No. of edges $E=m(n-1)+n(m-1)$.

If we compute any $F-E+V$, the result will be 1, which satisfies Equation (1)-i.e., the Euler histogram for planar objects. If we have $N$ objects superimposed by grid partitions, then this equation becomes:

$$
\begin{aligned}
\sum_{i=1}^{N} F_{i}-\sum_{i=1}^{N} E_{i}+\sum_{i=1}^{N} V_{i} & =(1+1+\ldots+1)-(1+1+\ldots+1)+(1+1+\ldots+1) \\
& =N-N+N \\
& =N .
\end{aligned}
$$

Therefore, the number of virtual objects is equal to $\min (\min (F), \min (E), \min (V))$. As demonstrated, each planar object (trajectory here, to be converted to a planar object) needs to have all face, edge and vertex counts computed correctly. Therefore, to compute the number of virtual objects, we need to have at least 1 face, 1 edge and 1 vertex count. In order to compute the number of virtual objects, we need to compute the minimum among these values to safely remove unnecessary counts related to virtual object(s) ( $c f$. Equation 4). However, when the row numbers or column numbers of a $\mathrm{QR}$ equal one, there will be only virtual lines. This implies that, there are only virtual faces and edges inside the $\mathrm{QR}$ and we need to find the minimum value of face and edge counts to compute the number of unnecessary virtual objects. As a result, the total number of objects, $T$, will be computed as: $T=N-N^{\prime}$. These rules are each represented in the $\mathrm{CASE}^{+}$algorithm.

Example 10 We demonstrate our technique via a detailed example. Figure 12 shows the procedure to compute the count for Figure 11. As it is depicted in Figure 11, we have 3 objects inside the whole area (partitioned to 6 by 8 grid cells). One of the objects (T1) is completely inside the $Q R$, the second (T3) is partially inside the $Q R$ and the last one (T2) is not inside the QR at all. However, since all three trajectories are causing the distinct counting problem, we need to planarize them into convex objects. Therefore, we will definitely have some virtual counts which are not related to any object inside the $Q R$ (and coming from T2). Based on the aforementioned rule, we compute the total number of objects inside the $Q R$ by considering real and virtual counts, CASE Equation (3). Then, we compute the number of unnecessary virtual objects as $N^{\prime}$ and eventually the total number of objects, $T$, via Equation (4). Specifically, in Figure 11, the QR has values $m=4, n=3$. The number of objects $N=\left(F+F^{\prime}\right)-\left(E+E^{\prime}\right)+V^{\prime}=(13+17)-(11+30)+14=3$; per each virtual face, virtual edge and virtual vertex, we have at least 1 as their values, therefore, we can deduct 1 from all of them to extract one virtual object; i.e., the minimum value for virtual histograms is 1 . That is, $N^{\prime}=\min \left(\min \left(F^{\prime}\right), \min \left(E^{\prime}\right), \min \left(V^{\prime}\right)\right)=(1,1,1)=1$. And the total number of objects $T=N-N^{\prime}=3-1=2$ is now corrected.

\subsubsection{Summary}

In a nutshell, some virtual counts are not necessary and can potentially yield errors for small query regions. $\mathrm{CASE}^{+}$is proposed to detect this source of error and rule out unnecessary virtual counts related to those objects, which are outside the $\mathrm{QR}$, but their virtual counts are inside. As depicted in Figure 12, all the real or virtual counts related to the provided example in Figure 11 are computed. Then, based on the proposed $\mathrm{CASE}^{+}$algorithm ( $c f$. 


$$
\begin{aligned}
& \text { Real Face Counts } \\
& \mathrm{F}[34]=1, \mathrm{~F}[35]=1, \mathrm{~F}[36]=1 \\
& \mathrm{~F}[44]=2, \mathrm{~F}[45]=1, \mathrm{~F}[46]=1 \\
& \mathrm{~F}[54]=1, \mathrm{~F}[55]=1, \mathrm{~F}[56]=1 \\
& \mathrm{~F}[64]=1, \mathrm{~F}[65]=1, \mathrm{~F}[66]=1
\end{aligned}
$$

Real Vertical Edges Counts

$\mathrm{E}[34-35]=1, \mathrm{E}[35-36]=1$

$\mathrm{E}[44-45]=1, \mathrm{E}[45-46]=0$

$\mathrm{E}[54-55]=0, \mathrm{E}[55-56]=0$

$\mathrm{E}[64-65]=0, \mathrm{E}[65-66]=0$

Real Horizontal Edges Counts

$\mathrm{E}[34-44]=1, \mathrm{E}[35-45]=0$

$\mathrm{E}[36-46]=1, \mathrm{E}[44-54]=1$

$\mathrm{E}[45-55]=1, \mathrm{E}[46-56]=1$

$\mathrm{E}[54-64]=1, \mathrm{E}[55-65]=1$

$\mathrm{E}[56-66]=1$
Virtual Face Counts

$\mathrm{V} \_\mathrm{F}[34]=1, \mathrm{~V} \_\mathrm{F}[35]=1, \mathrm{~V} \_\mathrm{F}[36]=1$

$\mathrm{V} \_\mathrm{F}[44]=1, \mathrm{~V}_{-} \mathrm{F}[45]=2, \mathrm{~V}$ F[46] $=1$

$\mathrm{V}_{-} \mathrm{F}[54]=2, \mathrm{~V}_{-} \mathrm{F}[55]=2, \mathrm{~V}_{-} \mathrm{F}[56]=1$

$\mathrm{V}_{-} \mathrm{F}[64]=2, \mathrm{~V}_{-} \mathrm{F}[65]=2, \mathrm{~V}_{-} \mathrm{F}[66]=1$

Virtual Vertical Edges Counts

$\mathrm{V} \_\mathrm{E}[34-35]=1, \mathrm{~V} \_\mathrm{E}[35-36]=1$

$\mathrm{V} \_\mathrm{E}[44-45]=2, \mathrm{~V} \_\mathrm{E}[45-46]=2$

$\mathrm{V}-\mathrm{E}[54-55]=3, \mathrm{~V}^{-} \mathrm{E}[55-56]=2$

$\mathrm{V} \_\mathrm{E}[64-65]=3, \mathrm{~V} \_\mathrm{E}[65-66]=2$

Virtual Horizontal Edges Counts

$\mathrm{V} E \mathrm{E}[34-44]=1, \mathrm{~V} E[35-45]=2$

$\mathrm{V}-\mathrm{E}[36-46]=1, \mathrm{~V}^{-} \mathrm{E}[44-54]=2$

$\mathrm{VE}[45-55]=2, \mathrm{~V} \mathrm{E}[46-56]=1$

$\mathrm{V} \_\mathrm{E}[54-64]=2, \mathrm{~V} E \mathrm{E}[55-65]=2$

$\mathrm{V}_{-} \mathrm{E}[56-66]=1$

Vertex Counts (always virtual); naming based on Z-order, row by row

$\mathrm{V}\left[34-35 \_44-45\right]=2, \mathrm{~V}\left[35-36 \_45-46\right]=2$

$\mathrm{V}\left[44-45 \_54-55\right]=3, \mathrm{~V}\left[45-46 \_55-56\right]=2$

$\mathrm{V}\left[54-55 \_64-65\right]=3, \mathrm{~V}\left[55-56 \_65-66\right]=2$

Fig. 12 Detailed computation of real and virtual face/edge/vertex counts for Figure 11.

Algorithm 3), minimum value of each virtual histogram can be extracted, [lines $1-3$ ]. Consequently, the number of virtual lines/objects, which can be deducted from the total counts, are calculated, [lines $4-8$ ].

Example 11 Other cases are possible, as demonstrated in Figure 13, in which an object is outside the QR but its virtual lines (counts) are not fully covered by the $Q R$, e.g., trajectory T4. In order to get perfect accuracy for the smaller QRs, these kinds of virtual objects must be removed as well. However, since we do not keep any identification to distinguish between trajectories, there is no guarantee that we can completely detect all these unnecessary virtual counts.

In our experimental study we implement $\mathrm{CASE}^{+}$and demonstrate improvements over CASE (and DEH), especially under smaller QRs where vanilla CASE can multiple count. 


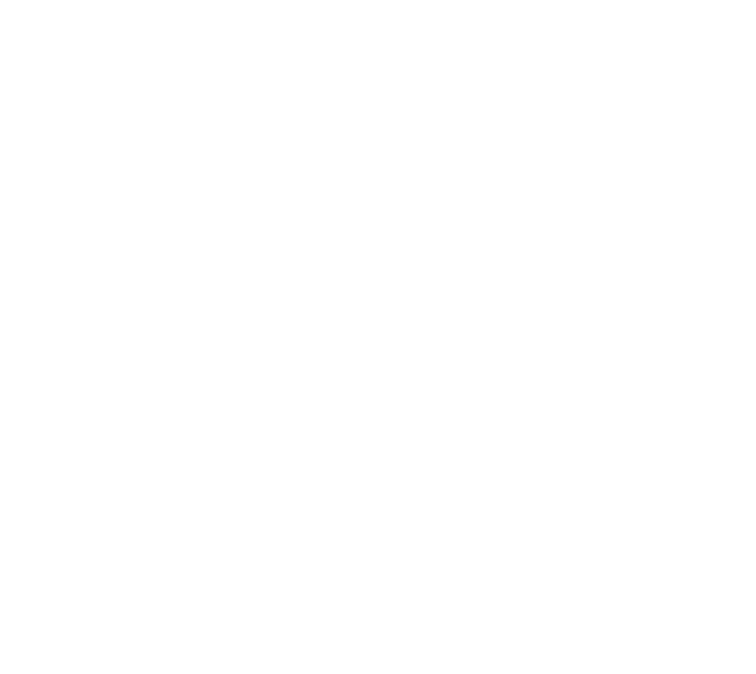

Fig. 13 Virtual counts for smaller QRs in CASE histograms may cause additional counts. QR does not fully cover the virtual counts related to $\mathrm{T} 4$.

\section{Privacy Analysis}

A goal of this paper is to preserve the privacy of individuals while releasing aggregate statistics. In this section, we quantify the ambiguity in reconstructing trajectories from count histograms, thereby bounding the privacy of our approaches. After describing our privacy model in Section 6.1, we present in Section 6.2 two worst-case settings where we enumerate the number of reconstructions possible from small histograms - it is exactly these histograms that pose the greatest risk to privacy. We then establish bounds on the privacy of more general histograms and consider the effect of spatial and temporal aggregation, in Sections 6.3 and 6.4. In Section 6.5, we explore the combination of Euler histograms with the Laplace additive noise mechanism of differential privacy. Our results bounds hold for all existing Euler histogram-based approaches $[3,37,47,48]$. We provide the first formal privacy analysis in this line of work.

\subsection{Privacy Model}

In our privacy analysis we consider the general setting of histogram-based counting of objects or trajectories. Referring to Figure 1, we consider two related models.

\section{- Service provider sanitizes}

- Users transmit their trajectories (or observed objects) to a trusted service provider.

- The service provider processes each incoming datum into increments of its stored histogram counts relative to a fixed partition-the trajectories/objects are never stored by the 3rd party, nor are their IDs stored in any form. The service provider makes public the histogram counts. 
- 3rd parties have full access to the histogram counts, and full knowledge of the service provider's mechanism including the partition used (following Kerckhoffs's principle [1])

- User sanitizes

- Users process their own trajectories (or observed objects) into increments to the histogram counts, and transmit only these increments. Users have possession of the partition to perform this computation.

- The service provider simply adds the delta's to their stored histograms, and publishes as above.

- 3rd parties act as above.

Non-interactive vs interactive privacy. Two privacy models have been studied for releasing datasets or their statistics: the interactive and non-interactive models [14]. In the noninteractive model, the database is sanitized and then released while the interactive model considers mechanisms that respond to queries by releasing approximate query responses. Our CASE-based approaches are naturally non-interactive, as we release the entire set of histograms. However, given a non-interactive mechanism it is trivial to derive an interactive mechanism: our architecture could instead require 3rd parties to submit query regions which the service provider responds to-we explore this scenario in Section 6.5.

Background knowledge and strength of privacy guarantee. Our target applications require only the occasional release of data to a traffic authority. Therefore we have implicitly assumed that the data gathering period of a dataset is significantly longer than the duration of individuals' trips, and that cells are sufficiently large to encompass multiple users. With sufficiently coarse spatio-temporal partitioning, correlations between collected datasets can be reduced. This significantly mitigates the level of background knowledge possessed by an attacker, and so limits the likelihood of successful location inference attacks [18]. It is well known that histograms are a natural approach to aggregation for preserving privacy [9]. In Section 6.5 we consider settings where stronger guarantees of privacy are required, e.g., if temporal or spatial aggregation is too fine-grained, if the attacker has background knowledge, or if the users collude maliciously. We demonstrate that Euler-histogram-based approaches are complementary to differential privacy, which is a general family of techniques guaranteeing that released statistics themselves do not breach privacy.

Pan privacy. While both of our above models retain no individual information, e.g., trajectories/objects or IDs, the service provider sanitizes setting must hold trajectories in memory temporarily. From the user's point of view, they must trust the service provider, as the provider may not actually remove the trajectory. There is no need for the submitted trajectory to be accompanied by any form of ID-e.g., the trajectory could be submitted anonymously via Tor [12]. Our second user sanitizes model eliminates this weakness by having the user herself process the trajectory, so that the service provider is never granted access to the raw data. As a result, even if an attacker had complete access to the service provider (e.g., if the provider were audited by a government), then no additional information is leaked other than what is published already. Therefore the second model is pan private [15].

To quantify the level of privacy preserved by histograms we propose the following notion, which formalizes the idea that reconstructing correct trajectories from counts should be difficult. 
Definition 3 Define a possibility as a multi-set of trajectories and objects in space, and let $h(P)$ represent the histogram induced by possibility $P$. Given histogram counts $H$, we define the confusion level $\mathscr{C}(H)$ of the histogram as the number of possibilities that induce histogram $H$, i.e., $\mathscr{C}(H)=\left|h^{-1}(H)\right|$.

Intuitively, $\mathscr{C}(H)$ represents the ambiguity inherent in corresponding possibilities with a histogram; the more possibilities that map to a histogram, the more privacy is preserved by that histogram. This definition applies to all existing approaches based on Euler histograms.

\subsection{Privacy in Worst-Case Settings}

We first consider the confusion level of a specific scenario where histogram counts are exceedingly small. Such settings represent the worst-case for the defender (the users and service provider): when counts are small there is little to no aggregation occurring. Understanding such settings provides a handle on worst case privacy. In Section 6.3 we establish a theoretical result that can lift the calculations of confusion level of this section to larger histograms. In Section 6.5 we discuss strategies for dealing with these worst cases.

\subsubsection{Enumerating Trajectory Types}

Before exploring the worst-case scenario, we build up a complete enumeration of trajectory types. We consider a grid of $m$ rows and $n$ columns ( $c f$. Figure 14), where a cell $(i, j)$ has four neighbouring cells — at its North, South, West and East sides, denoted $N, S, W, E$-and four edges in common with these neighbouring cells.

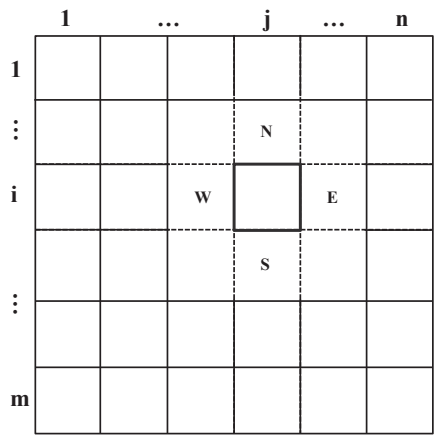

Fig. 14 Typical grid, cell and cell neighbours

To build up our understanding of the possible types of trajectories, we focus on a single grid cell. In Figure 15, we show all the possible cases that can occur for cell $(i, j)$ in terms of a trajectory's path, direction and origin/destination. The subset of possible cases depends on histograms of the cells surrounding cell $(i, j)$.

For trajectories originating/ending in the cell, there are 4 possible undirected cases and 8 possible directed cases ( $c f$. Figure 15a). For trajectories passing through the cell without turning, there are 2 and 4 cases respectively ( $c f$. Figure 15b). Finally, there are 4 and 8 cases respectively, of paths that pass through the cell and turn ( $c f$. Figure 15c). In general, direction doubles the number of cases, and is the typical default for road network applications. 

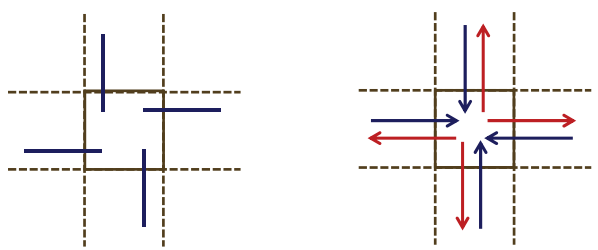

(a) A trajectory path which may end or start.

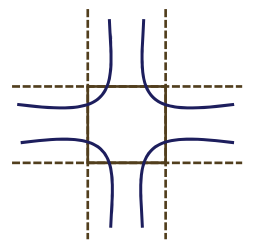

(c) A trajectory path that turns by passing the cell.
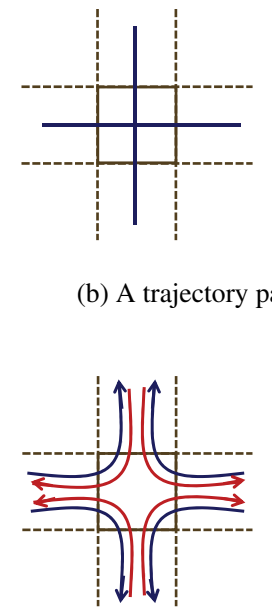

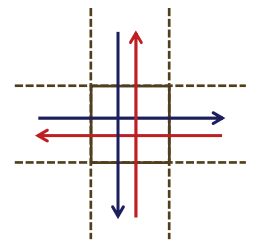

(b) A trajectory path which passes the cell.

Fig. 15 All the possible cases for a trajectory path that can occur through a cell.

When analysing a histogram for privacy, we wish to count the number of possibilities that lead to the histogram counts. In general this confusion level is increased when trajectories can be bidirectional (multiple trajectories lead to exponentially more possibilities), or when the histogram counts are large.

\subsubsection{A Worst-Case Histogram on the Line}

The simplest case for the attacker to track a trajectory is to have $m=1$ (a linear grid) with all edge and face counts equal to 1 . If trajectories are undirected then there is only one possibility inducing this histogram: a single trajectory passing through each cell. With direction, the confusion level becomes 2 .

While this is an absolute worst-case, it is similar to having a cell with count 1 surrounded by zero cells, in a 2-dimensional grid. The former linear grid is somewhat contrived since applications tend to involve 2-dimensional grids; we address the latter case in Section 6.5.

\subsubsection{A Worst-Case Histogram in the Plane}

Consider now the case where the face count of cell $(i, j)$ is 2 , cells $N, W, E$ and $S$ have counts of 1 , the edges shared with cell $(i, j)$ have also counts of 1 , and all other counts are zero. As illustrated in Figure 16a, without considering the directions there are 3 possible reconstructions of trajectory paths. With two directions the confusion level grows quickly to 12 (cf. Figure 16b). Even with exceedingly small counts, the difficulty of reconstructing trajectories, as given by the confusion level, is high. 

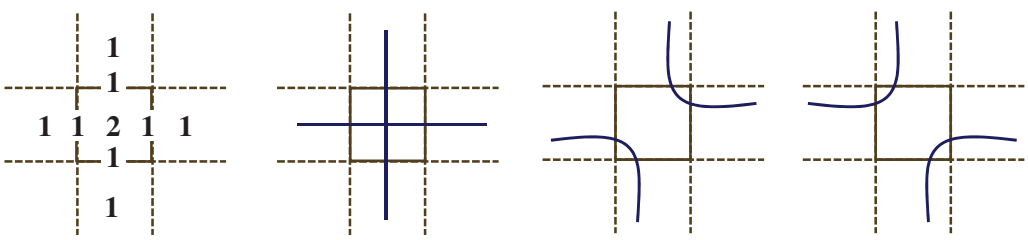

(a) A simple Euler histogram and the undirected possibilities that induce it.
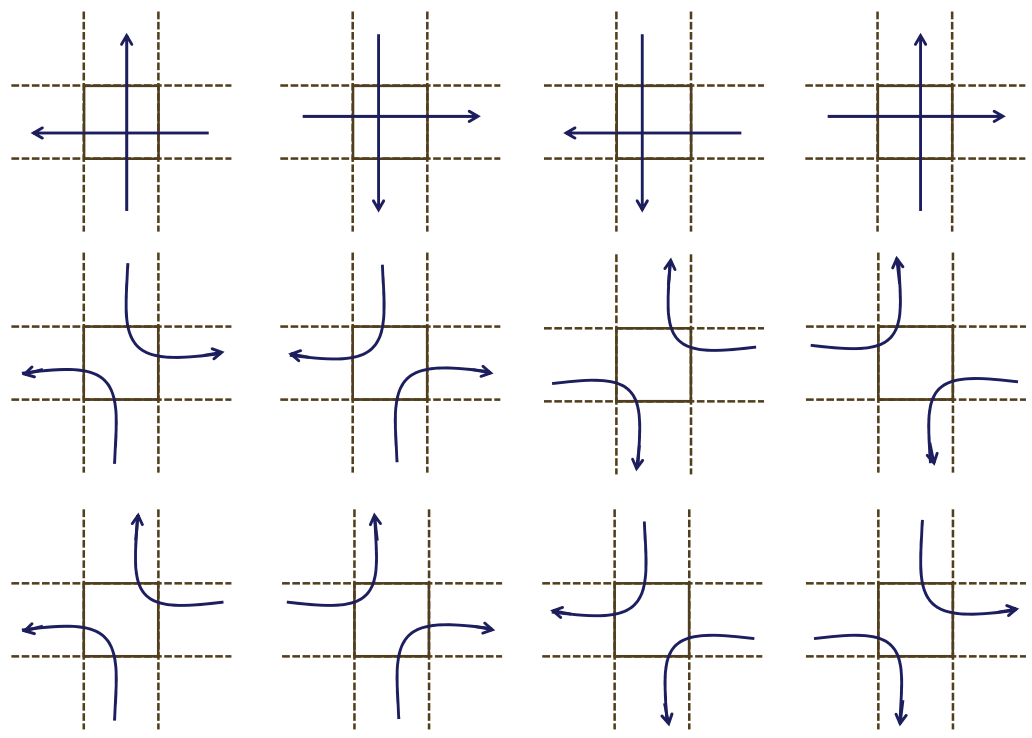

(b) The directed possibilities that induce the Euler histogram.

Fig. 16 A worst-case example with high confusion level.

\subsection{A Lower Bound on Privacy}

In general, the more counts we have, the more possibilities are feasible. The confusion of a histogram is at least linear in the counts, and it is expected to grow exponentially in some cases. We contribute the following theorem for any count histogram. Our result holds for histograms produced by any of the approaches based on Euler histograms $[3,37,47,48]$ as they all satisfy the following property.

Definition $4 A$ histogram construction algorithm $\mathscr{A}$ that maps possibilities to histograms is additive if for any possibilities $P, Q$ it holds that $\mathscr{A}(P \cup Q)=\mathscr{A}(P)+\mathscr{A}(Q)$, where + represents elementwise addition of the histogram counts and $\cup$ represents the multiset union of possibilities.

Theorem 1 For any histogram $H$ produced by an additive histogram construction algorithm, and natural number $k$, the histogram $k \cdot H$ formed by elementwise multiplication of the counts of $H$ by $k$, satisfies $\mathscr{C}(k \cdot H) \geq k \cdot \mathscr{C}(H)-(k-1)$.

Proof Let $P=h^{-1}(H)$ be the set of all possibilities with histogram $H$. Then by definition of confusion, $|P|=\mathscr{C}(H)$. Select any possibility $p_{0}$ from $P$, and construct a new set of 
possibilities

$$
P^{\prime}=\left\{p_{0} \cup p: p \in P, p \neq p_{0}\right\} \cup\{p \cup p: p \in P\} .
$$

Since for all $p, q \in P(H), p \neq q$ implies $p \cup p \neq q \cup q$, it follows that each choice of $p$ in the two components making up $P^{\prime}$ constructs distinct possibilities and so $\left|P^{\prime}\right|=2|P|-1$. By the additivity of the histogram construction algorithm, we have that $h(p)=2 \cdot H$ for all $p \in P^{\prime}$. In other words,

$$
2 \mathscr{C}(H)-1=\left|P^{\prime}\right| \leq \mathscr{C}(2 \cdot H)
$$

This proves the case for $k=2$.

The case for general $k>2$ is similar: the first component of $P^{\prime}$ is now made by taking unions of $k$ possibilities total, with some number $d$ of repeats of $p_{0}$ and $k-d$ many $p \neq p_{0}$. Each of these constructed possibilities is distinct as before, and induces the desired $k \cdot H$ histogram. The second component making up $P^{\prime}$ is formed by simply taking $k$ th powers of each possibility $p \in P$. In particular if we define $p^{d}=\bigcup_{i=1}^{d} p$ for any $p \in P$ we have

$$
P^{\prime}=\left\{p_{0}^{d} \cup p^{k-d}: p \in P, p \neq p_{0}, d \in\{1, \ldots, k-1\}\right\} \cup\left\{p^{k}: p \in P\right\} .
$$

Again, we have that $h(p)=k \cdot H$ for all $p \in P^{\prime}$, and now $k \cdot \mathscr{C}(H)-(k-1)=\left|P^{\prime}\right| \leq \mathscr{C}(k \cdot H)$.

This result demonstrates that larger counts lead to more confusion and greater privacy. In particular, we can apply the bound to generalise our worst-case examples.

Corollary 1 Let $H_{k}$ be a histogram with face count of cell $(i, j)$ equals to $2 k$, cells $\mathrm{N}, \mathrm{W}, \mathrm{E}$ and $\mathrm{S}$ of $(i, j)$ having counts of $k$, the edges shared with cell $(i, j)$ having counts $k$, and with all other counts zero. Then $\mathscr{C}(H)$ is at least $11 k+1$ for directed trajectories or $2 k+1$ for undirected trajectories.

\subsection{The Effect of Aggregation on Privacy}

It is intuitively clear that larger confusion is achieved by increasing aggregation, either in the temporal or spatial dimensions. We quantify this effect, for simple settings that apply for all existing Euler histogram-based approaches, which are all additive.

First for increasing temporal aggregation, we have immediately from additivity:

Lemma 1 Consider any temporal partition $\left[t_{0}, t_{1}\right),\left[t_{1}, t_{2}\right), \ldots$, where $t_{i+1}>t_{i}$, and histograms $H_{i}$ computed in each period $\left[t_{i-1}, t_{i}\right)$ by an additive histogram construction algorithm. Then for any $i$, the algorithm run over the aggregate interval $\left[t_{i-1}, t_{i+1}\right)$ would have produced the histogram $H_{i}+H_{i+1}$.

From this and Theorem 1 it follows that

Corollary 2 Consider the same setting as Lemma 1 . If for any $i$, histograms $H_{i}=H_{i+1}$ then the histogram produced over aggregate interval $\left[t_{i-1}, t_{i+1}\right)$ is $2 \cdot H_{i}$, and has confusion level at least $2 \cdot \mathscr{C}\left(H_{i}\right)-1$.

And from the lemma and linearity of expectation we have 
Corollary 3 Consider the same setting as Lemma 1, where possibility $p_{i}$ observed in time interval $\left[t_{i-1}, t_{i}\right)$ is random according to some distribution. Where $p_{i} \cup p_{i+1}$ represents the possibility recorded for the aggregated interval $\left[t_{i-1}, t_{i+1}\right)$, then the expectation ${ }^{1} \mathbb{E}\left[h\left(p_{i} \cup\right.\right.$ $\left.\left.p_{i+1}\right)\right]=\mathbb{E}\left[h\left(p_{i}\right)\right]+\mathbb{E}\left[h\left(p_{i+1}\right)\right]$. In particular, if both intervals have expected histogram $\mathbb{H}$ then $\mathbb{E}\left[h\left(p_{i} \cup p_{i+1}\right)\right]=2 \cdot \mathbb{H}$.

Roughly speaking, doubling time of data collection yields double the histogram counts, and increased privacy due to the increased confusion level. We can also analyse the effect of increasing spatial aggregation in an idealised setting.

Definition 5 We call an unbounded partition regular if all face counts are identically $f$, all edge counts are identically $e$, and all vertex counts are identically $v$, for some $f, e, v$.

We have chosen to define regular partitions as unbounded, so as to simplify the feasibility of taking identical face counts (and also edge, vertex counts) across cells. We use such symmetrical counts to simplify the discussion in the next proposition, however regularity is not necessary nor is unboundedness. Indeed the next result concerns only a local collection of cells.

Proposition 1 Consider a regular partition with counts $f, e, v$ obtained from observing convex bodies, Figure 17-whereby all Euler histogram derived approaches calculate objects correctly via Equation (1). If all $2 \times 2$ groups of cells are aggregated into a new partition of larger cells, then the face, edge, vertex counts obtained from rerunning histogram construction with the new partition become

$$
\begin{aligned}
f^{\prime} & =4 f-4 e+v \\
e^{\prime} & =2 e-v \\
v^{\prime} & =v
\end{aligned}
$$

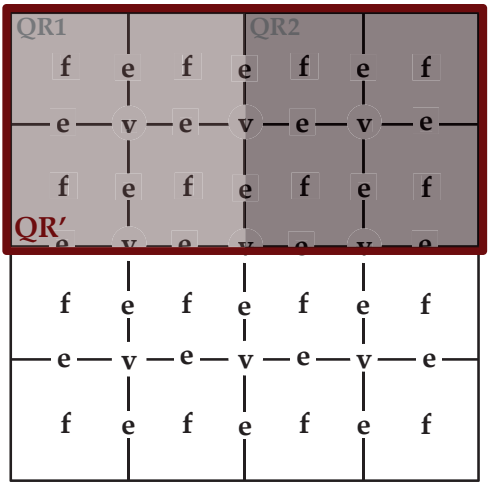

Fig. 17 A regular partition with counts $f, e, v$. Region $Q R^{\prime}$ is the aggregation of $Q R 1$ and $Q R 2$.

\footnotetext{
1 This expectation is taken with respect to the random possibilities.
} 
Proof The proof involves two applications of Equation (1) for estimating $f^{\prime}$ and $e^{\prime}$. Consider a $2 \times 2$ region $Q R 1$ that covers a $2 \times 2$ block of cells. The number of objects in $Q R 1$ is exactly $4 f-4 e+v$, and this number corresponds to the face count $f^{\prime}$ of the new $2 \times 2$ aggregate cell establishing the first part. Consider again $2 \times 2 Q R 1$, a neighbouring $2 \times 2 Q R 2$, and finally $Q R^{\prime}$ that is the $4 \times 2$ union of $Q R 1$ and $Q R 2$. By an inclusion-exclusion argument, it is easy to see that $\left|Q R^{\prime}\right|=|Q R 1|+|Q R 2|-|Q R 1 \cap Q R 2|$ where $|\cdot|$ refers to the object count and the intersection term corresponds to the count of objects lying in both regions. Then $\left|Q R^{\prime}\right|$ can be found by a second application of Equation (1) to be $8 f-10 e+3 v$. Noting that $|Q R 1 \cap Q R 2|=e^{\prime}$, and that $|Q R 1|+|Q R 2|=2 f^{\prime}$, we can solve for $e^{\prime}$ to yield $e^{\prime}=2 e-v$. Finally regarding a vertex in the new partition, it is clear that its count remains the same.

Intuitively this spatial aggregation result is similar in spirit to the temporal results: larger cells can result in larger histograms (and therefore also privacy). The degree to which histograms grow, depends on the relative size of observed objects compared to grid cells. More trajectories that span multiple cells implies higher edge counts, which dampen the growth of histograms when aggregating. Small trajectories mostly contained in cells implies small edge counts.

Example 12 Consider an idealised regular partition in which e $=4 v, f=8 v$. Proposition 1 yields that doubling the grid cell sizes leads to new counts $e^{\prime}=7 v, f^{\prime}=17 v$; an almost doubling.

\subsection{Protecting Against Background Knowledge: Differential Privacy}

While sufficient aggregation leads to high levels of privacy, pathological counts can reveal sensitive information.

\subsubsection{Sanitizing Specific Pathological Counts}

The case of a single face count of 1 with neighbouring edge counts of 0 may reveal information about a user living in a sparsely-populated area: e.g., an attacker may find that this observation in the specified time period, belongs to a private house through map-matching. A defence against such pathological cases is to test for their presence in the current state of histograms, and zero them out. For example, we could test for a face count of 1 with adjoining edge counts of 0 , and set the face count to 0 (or refuse to release the count at all).

\subsubsection{Differential Privacy}

More generally, the service provider may be required to guarantee a strong notion of privacy: that the released counts reveal nothing significant about users not already known to a powerful attacker. Such a requirement is the focus of differential privacy [14].

Definition 6 Let $D$ be a database of individuals' records. We say that $D$ and another database $D^{\prime}$ are neighbours if they differ on exactly one record. If $\mathscr{T}$ denotes the space of possible statistics that could be released to summarise aspects of $D$, then a mapping $\mathscr{M}: D \mapsto t \in \mathscr{T}$ that outputs such a response given data is called a mechanism. Mechanisms can be either deterministic, or randomised (outputting each possible response $t \in \mathscr{T}$ with some probability). 
Definition 7 Let $\varepsilon$ be a positive real, and $\mathscr{M}$ some randomised mechanism. If for all neighbouring databases $D, D^{\prime}$ and measurable sets of responses $T \subseteq \mathscr{T}$,

$$
\operatorname{Pr}(\mathscr{M}(D) \in T) \leq \exp (\varepsilon) \cdot \operatorname{Pr}\left(\mathscr{M}\left(D^{\prime}\right) \in T\right),
$$

then we say that $\mathscr{M}$ preserves $\varepsilon$-differential privacy.

Differential privacy exactly states that the mechanism's response distributions on $D$ and $D^{\prime}$ are pointwise, multiplicatively, close: the probability of outputting each response is insignificantly influenced by the input data. The measure of closeness is given by parameter $\varepsilon$. Smaller $\varepsilon$, the greater the level of insensitivity of the mechanism to small perturbations to the data.

To understand why differential privacy provides a strong, semantic notion of privacy, we may consider a powerful attacker wishing to uncover the last record in true database $D$, given

- Complete knowledge of $\mathscr{M}$ up to randomness;

- Access to a number of responses sampled from $\mathscr{M}(D)$, limited to $O(|D|)$;

- Knowledge of $D$ except for the target record; and

- Unlimited computational resources.

Such an omnipotent and omniscient attacker could

1. Query $\mathscr{M}(D)$ a total of $O(|D|)$ times to construct a histogram $\hat{P}$ approximating the true distribution $P(D)$ of $\mathscr{M}(D)$;

2. Simulate all possible databases $D^{\prime}$ by filling in the missing record. For each:

(a) Simulate the exact distribution $P\left(D^{\prime}\right)$ and compare it with $\hat{P}$

3. Output the $D^{\prime}$ with $P\left(D^{\prime}\right)$ most closely matching $\hat{P}$.

Even against this incredibly powerful attack, differential privacy guarantees that all the $P\left(D^{\prime}\right)$ are exceedingly close to each other, and in particular to the true $P(D)$. Since the number of queries to the mechanism is limited, the estimation error of $\hat{P}$ approximating $P(D)$ is larger than the distances between the $P\left(D^{\prime}\right)$ s, for sufficiently small $\varepsilon$. As a result it is impossible for the attacker to distinguish which database $D$ induced the mechanism's responses.

Put another way, an attacker can not detect arbitrary changes of a single datum made by a user; or indeed, could not detect if the user removes their datum altogether.

\subsubsection{Global Sensitivity and the Laplace Mechanism}

We now introduce a popular approach to converting deterministic mechanisms into randomised mechanisms that preserve differential privacy. First we provide a definition that quantifies the influence of data perturbations to a mechanism's output.

Definition 8 We say that a deterministic mechanism $\mathscr{M}$ has $L_{1}$-global sensitivity $\Delta>0$ if for every pair of neighbouring databases $D, D^{\prime}$ we have $\left|\mathscr{M}(D)-\mathscr{M}\left(D^{\prime}\right)\right| \leq \Delta$.

This definition generalises to vector-valued mechanisms, in which case the difference between responses is measured by the $L_{1}$ norm which generalises absolute distance. Observing that under the hamming distance, the distance between neighbouring databases is 1, it follows that global sensitivity corresponds to a Lipschitz condition on the mechanism with $L_{1}$-norm in the response space and hamming (or $L_{1}$-norm) in the input database space. 
Lemma 2 ([14]) For any $\varepsilon>0$, if deterministic mechanism $\mathscr{M}$ has $L_{1}$-global sensitivity $\Delta>0$, then randomised Laplace mechanism given by

$$
\mathscr{M}^{\prime}(D)=\mathscr{M}(D)+Z,
$$

where $Z$ is distributed as zero-mean Laplace noise with scale $\Delta / \varepsilon$, preserves $\varepsilon$-differential privacy.

The proof is a straightforward application of the triangle inequality, with the definition of the Laplace pdf, and the definition of global sensitivity. The power of this mechanism and others in the differential privacy literature, is that provided global sensitivity can be measured, then we immediately have an applicable mechanism whose differential privacy is already established.

\subsubsection{Differentially-Private Range Queries}

We now explore the combination of differential privacy with Euler histogram based approaches such as CASE. While a non-interactive model that releases the entire histogram may be possible [2], we focus on an interactive model in which a 3rd party queries the service provider with a specific $\mathrm{QR}$. Under such a model, we have the following differentiallyprivate mechanism

Theorem 2 For a given query region $R$, let $N(R)$ denote an object count calculated from the current histogram. Then the randomised interactive mechanism $N_{D P}(R)=N(R)+$ Laplace $(0,1 / \varepsilon)$ is $\varepsilon$-differentially private for any $\varepsilon>0$.

Proof The result follows from the global sensitivity of a count being 1: if a single entry of the database (a trajectory) is perturbed, the count $N(R)$ can change by at most 1 . Together with Lemma 2 the result follows.

This result demonstrates that differential privacy and Euler histograms are not at odds and indeed complement each other. While we do not consider $N_{D P}(\cdot)$ further in this paper, future work could focus on non-interactive mechanisms.

\section{Setting Grid and Query Region Sizes}

Grid cell size can affect accuracy as well as privacy: the larger the grid cell size, the smaller the amount of double-counting and granularity of counts possible, and the higher the level of privacy. Selecting grid cell size can be accomplished in several ways, here we explore one possible approach that a traffic authority might follow.

Initially the authority may have the following known

- The bounding dimensions of the city to be analysed, we assume without loss of generality to be $n \times n \mathrm{~km}^{2}$;

- The number of road links in the city $L$; and

- A target average number of links per cell $A$.

The average links per cell could come from discussions or policy on privacy, and links could be replaced by population density without any change to the algorithm for setting grid size. Once the authority selects a grid size for one city, perhaps arbitrarily, they may also want 
to achieve the same average links per cell in subsequent cities, in which case the following process would apply also.

An efficient process for selecting square cell size length $m(\mathrm{~km})$ is the following binary search:

1. Initialise cell size $m$;

2. Initialise achieved links-per-cell $a \gg A$

3. Repeat until $a \approx A$ :

(a) Calculate achieved links-per-cell $a$ for current $m$

(b) If $a<A$ double $m$; otherwise halve $m$

The initial grid size $m$ can be estimated assuming that links are evenly distributed across the city. For a given grid size $m$, the number of cells $C$ in the partition created corresponds to

$$
C=\left\lceil\frac{n}{m}\right\rceil^{2}
$$

And noting that the average links-per-cell achieved under the uniformity assumption is $L / C$, we can set this fraction to be equal to target $A$, and solve for $m$ :

$$
\begin{aligned}
A & =L / C=L /\left(\frac{n}{m}\right)^{2} \\
\Leftrightarrow m & =n \sqrt{\frac{A}{L}} .
\end{aligned}
$$

Example 13 For example consider Beijing, targeting $A=6$ links per cell, city dimensions of $n=20 \mathrm{~km}$ squared, and total number of links $L=57,553$. Then the initial grid size $m$ is estimated at $204.2(\mathrm{~m})$ which rounds to $200(\mathrm{~m})$.

It is shown in the experimental study, Section 8, how $m$ affects the level of accuracy especially for smaller QRs e.g., when the grid cell size $m=1 \% \times \mathrm{QR}$.

Theoretically, we would expect that when the query rectangle size increases so too does the accuracy. However, the shape of the query rectangle also affects accuracy, e.g., when the $\mathrm{QR}$ is thin or is not a square. For this reason we sometimes see inconsistencies of increasing accuracy for smaller QRs. In our experimental study, we explore a variety of QR shapes included in an average over accuracy results. To further study diverse QR, we randomise QR positions.

Larger QRs are applicable in several cases. For instance, the Beijing road network consists of several rectangular-shaped ring roads around the same centre. These areas have been defined as the city's inner, middle, and outer areas based on the rings. Therefore, the Beijing traffic authority may want to run queries on correspondingly large rectangular areas. Similarly, in Melbourne, VicRoads collects the length of roads travelled by vehicles in three zones (inner, middle, outer). Each of the zones is quite a large area. In terms of QR size, our algorithm provides $100 \%$ accuracy for larger QRs experimentally and a high level of accuracy for smaller ones through the use of virtual counts.

\section{Experimental Study}

We perform experiments on the road networks of three major cities, Melbourne $10 \mathrm{~km} \times$ $10 \mathrm{~km}$, Manhattan $4.7 \mathrm{~km} \times 14.7 \mathrm{~km}$, and Beijing $20 \mathrm{~km} \times 20 \mathrm{~km}$ ( $c f$. Figures 18, 19 and 20). 
For the latter, we also experiment on a real dataset, T-Drive, in Section 8.6.1. For every city, we compute the initial size of the grid cell so that the number of links per grid cell remains approximately constant by applying Equation (5). We target $\approx 6$ links per cell. Consistent links per cell is natural in terms of privacy, and also leads to consistent accuracies across cities. According to this setting, the default (rounded) grid cell sizes for Melbourne, Manhattan and Beijing are 100, 150 and 200 meters respectively. These values could also be driven from the distance between road junctions on average, which is of interest to traffic authorities.

\subsection{Setting up the Environment}

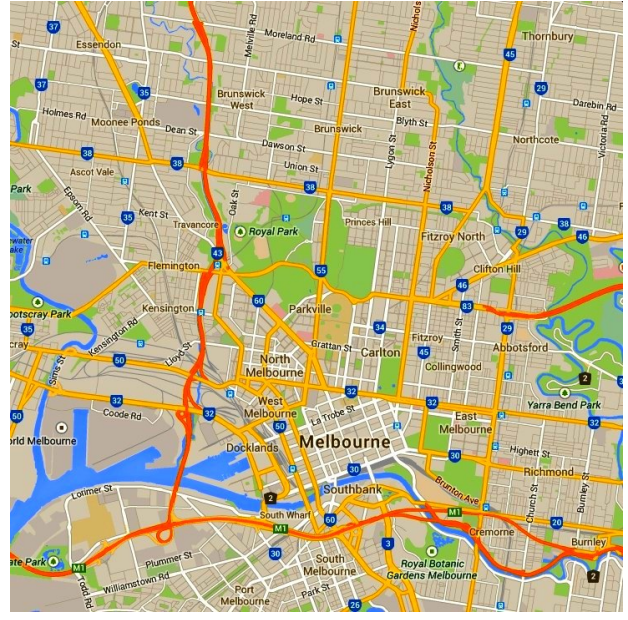

(a) Map of the selected area.

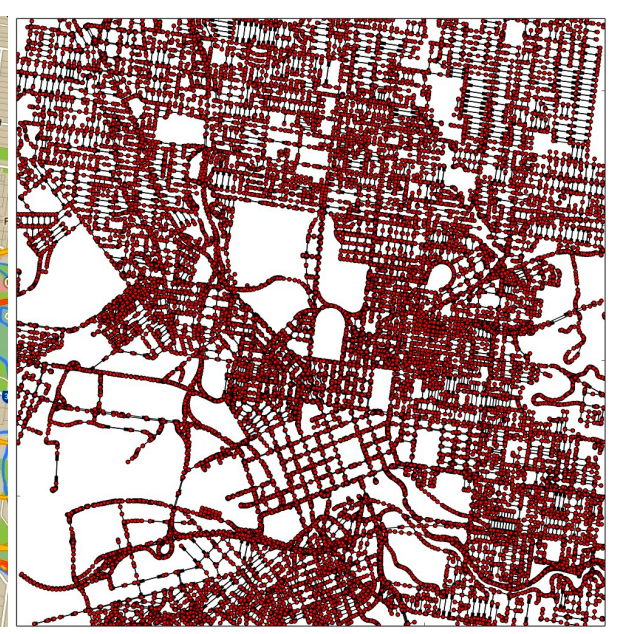

(b) Created graph of the selected area.

Fig. 18 Map and graph of the selected area of the city of Melbourne.

In order to set up the environment to run our experiments, we create moving object paths for the Melbourne, Manhattan and Beijing city road networks. We have generated our road network graph for a populated area of the Melbourne city by using real road data available from the OpenStreetMap (OSM) [28] website, which was also used for Manhattan and Beijing. We created a graph based on the node list and the adjacency list extracted by an OSM parser. We cleaned the extracted data in order to have a strongly connected graph. Our created graph is directed, i.e., we consider actual street directions. Figure 18a shows the selected area of the Melbourne city map of our experimental study. This area covers Melbourne's central business district (CBD) and surrounding suburbs that are the most populated (e.g., high traffic volume) areas of Melbourne. We then created the graph of this selected area ( $c f$. Figure 18b). Our pre-study parameter values are a grid cell size of 100 meters and the number of trips, $1000(1 \mathrm{k}), 5 \mathrm{k}$ and 10k. Figure 19 demonstrates Manhattan and Figure 20 depicts the selected area of Beijing together with their related graphs.

For each trajectory, we define various trip patterns on each road network. To create each trip we i.i.d. uniformly randomly select source and destination or destinations depending on 
the trip types ( $c f$. Section 8.2). We generate the shortest path between each pair of randomly selected points to create the trajectory, simulating a real road user. To prepare the experimental environment, in Sections 8.2 and 8.3 we discuss different trajectory trip patterns and baseline techniques. Section 8.4 shows the experimental results regarding many configurations of the parameters. We compare our two algorithms, $C A S E$ and $C A S E^{+}$, in Section 8.4 against the baseline methods.

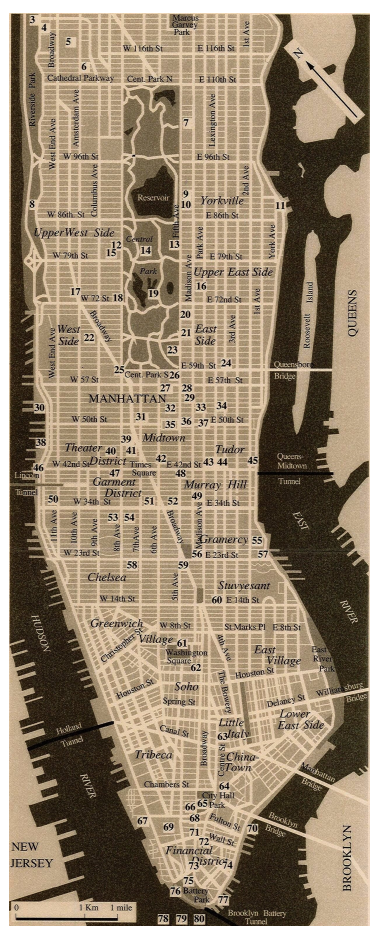

(a) Map of the area.

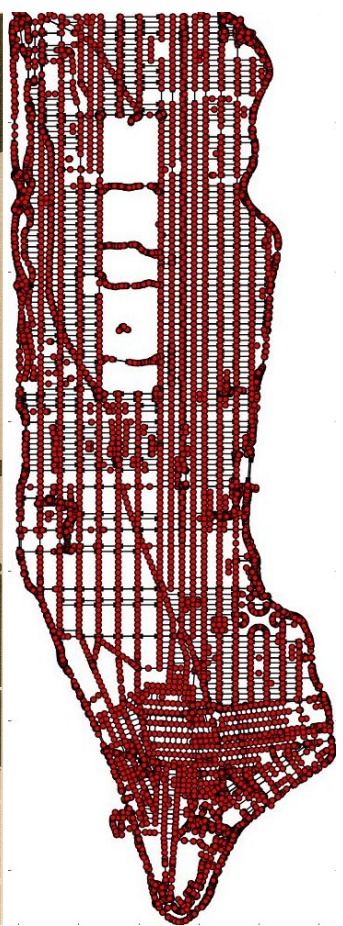

(b) Created graph of the area.

Fig. 19 Map and graph of the rotated Manhattan city.

As described in Section 3, there are a variety of trip patterns for moving objects. In the next section, we define typical trips happening during the day to set up our experimental study.

\subsection{Trajectory Trip Patterns}

Various types of trips result in different moving object trajectories. For instance, a user can start a journey from home to a shopping centre and then return back to home. In this case, there is one stop between the source and destination. There could be $k$ stops between a source and a destination in any created trajectory. Hence, we define some trip patterns created every day by moving objects. We will now discuss different trip types and especially those causing the distinct counting problem. 


\subsubsection{One-way Trip}

A one-way trip, is a single trip which has no stop en route, $k=0$. Therefore, we have only a source $(S)$ and a destination $(D)$. An example of this trip is travel from home to an airport.

\subsubsection{Return Trip}

As a special case of $k$-stop shortest path, we define a return trip when we have 1 or more stops between the source and the destination, $k \geqslant 1$. Therefore, if $k=1$ (one stop in between) implies $S, D 1$ and $D=S$ are the visited stops. If $k=2$ (two stops in between) implies $S, D 1$, $D 2$ and $D=S$ are the visited stops. A shopping trip is an example of this trip pattern which is travel from home to a shopping centre and then return back to home. A postal delivery could create this trip type as well, usually with more than one stop in between.

\subsubsection{Circling Trip with $n$-cycles}

In trips with $n$ cycles, a moving object starts a journey from a source and then after visiting at least four stops, $D 1, D 2, D 3, D 4$, it still needs to return back to the first stop and repeats its journey, $k \geqslant 4$. In a typical grid-aligned road network we need to have four stops to create a cycle, but technically three nodes can create a cycle in some road networks.

For example: $S, D 1, D 2, D 3, D 4, D 1, D 2, D 3, D 4, D 1, \ldots$ could be the visited stops. For instance, taxi cabs looking for customers or drivers looking for a parking space create trips with $n$ cycles.

Circling trips with $n$ cycles may lead to a reduced accuracy in the calculated number of the trajectories. We define them as follows: any set of stop points in which a trajectory revisits them more than once is called a cycle. The source and the destination of any cycle should be same and the other stop points will be revisited depending on the number of cycles. If the number of cycles equals to 1 , every stop point is visited twice.

The common feature in return trips and circling trips with $n$ cycles is the shape of these trips. The shape of the trajectories as created in these trip patterns are returning back to the source in return trips, or any other stops in circling trip.

\subsubsection{Mixed Trip}

For mixed trips, we assign a uniform portion for each of the above-mentioned trip patterns. In other words: $25 \%$ one-way trips, $25 \%$ return trips with 1 stop, $25 \%$ return trips with 2 stops and $25 \%$ trips with 1 cycle. However, in a realistic scenario, return trips have the higher possibility to occur.

In our experiments, we study three techniques as our baseline approaches ( $c f$. Section 8.3), to evaluate the CASE algorithm. We change various parameters such as query rectangle size, grid cell size, number of trips and type of trips to show that the CASE histogram can always achieve a high level of accuracy that often outperforms alternate approaches.

\subsection{Baseline Approaches}

In addition to Euler histograms and DEH [47], we have also implemented Euler ${ }^{\star}$ which is an enhanced version of the Euler histogram for moving object trajectories, so that it is a more fair comparable with CASE ( $c f$. Section 8.3.2 for details). 


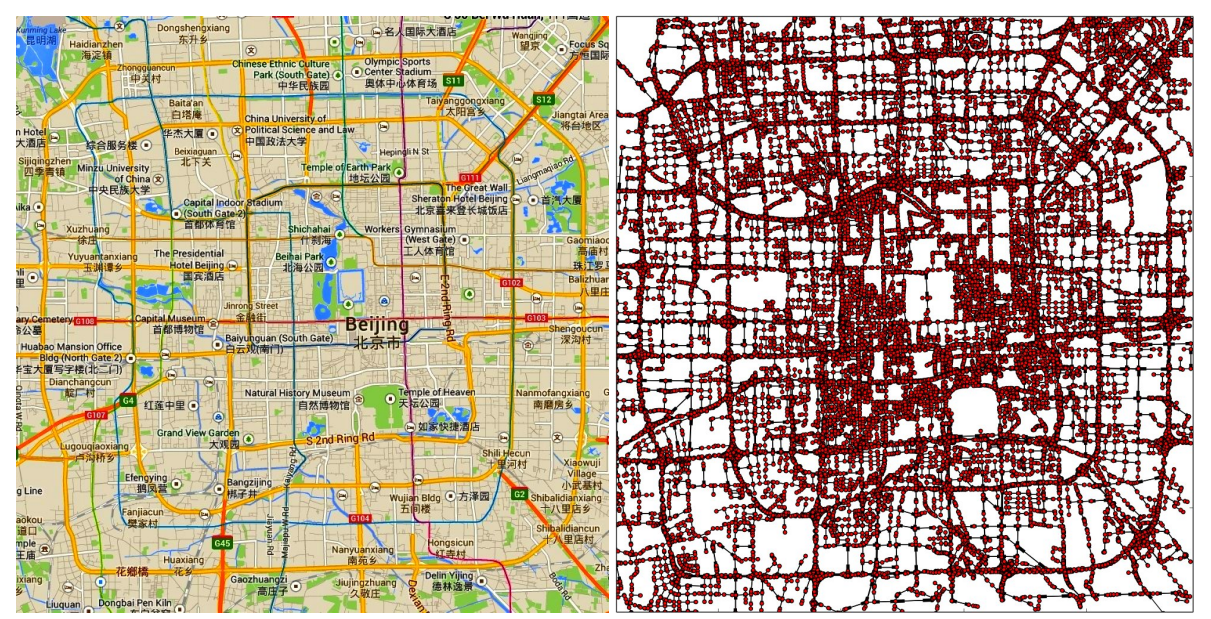

(a) Map of the selected area.

(b) Created graph of the selected area.

Fig. 20 Map and graph of the selected area of the city of Beijing.

\subsubsection{Euler/DEH}

In the Euler histogram, in order to compute the distinct count of the moving object trajectory as a list of node IDs, it is necessary to retrieve the related cell IDs and edge IDs of every trajectory link. Before appending the current cell ID to the list we check if two consecutive links are located in the same cell. If that is the case, this extracted cell ID is not added. Furthermore, two links which are consecutive could cross the same edge. Here, we need to consider only one edge ID in the extracted edge ID list. For all of the cell IDs and edge IDs in this extracted list, we will add one count to their related face and edge histograms. Finally, Equation (2) will be applied.

\subsubsection{Euler $^{\star}$}

To supply existing Euler-based approaches with the same information available to CASE histograms - so as to conduct a fair comparison-we extend DEH with an approach we term Euler ${ }^{\star}$. Euler ${ }^{\star}$, by contrast to DEH, considers the full trajectory in the first step to compute face and edge counts. This implies that when we have two counts in a cell that belong to the same trajectory, Euler ${ }^{\star}$ considers only one. Therefore, the technique receives every trajectory path as a list of node IDs, then retrieves the related cell IDs and edge IDs of every trajectory link. Here, if the current extracted cell ID exists in the cell ID list, it will not be re-added. This happens if two consecutive links are located in the same cell or a trajectory re-enters a cell. In both cases, the related cell ID should be considered only once. Whether an edge ID should be added to the edge ID list or not, depends on another rule which avoids repetition: if a cell has already been visited by the current trajectory, then the current edge ID is not considered. For all of the cell IDs and edge IDs in the extracted list, we will add one count to their related face and edge histograms. Finally, Equation (2) is applied. 


\subsubsection{SID}

We consider the Simple ID (SID) technique as a baseline providing an accurate answer to evaluate our approach. This method receives every trajectory path as a list of node IDs, then retrieves the related cell IDs of every link. Here, as above, the IDs in the cell ID list are unique. In these retrieved cells we keep the trajectory ID instead of the count. Thus, SID is always $100 \%$ accurate.

Table 1 Experimental settings for Melbourne City with respect to Grid Cell Size, No. of Trips, Trip Types, and QR Size. In each row, the bolded items are varied with the non-bolded items are held fixed.

\begin{tabular}{|c|c|c|c|c|}
\hline & Grid Cell Size & No. of Trips & Trip Types & QR Size \\
\hline 1 & $100 \mathrm{~m}$ & $1 \mathrm{~K}$ & One-way (O) & $1-10 \% 10-100 \%$ \\
\hline 2 & $100 \mathrm{~m}$ & $1 \mathrm{~K}$ & Return 1-Stop (R1) & $1-10 \% 10-100 \%$ \\
\hline 3 & $100 \mathrm{~m}$ & $1 \mathrm{~K}$ & Return 2-Stops (R2) & $1-10 \% 10-100 \%$ \\
\hline 4 & $100 \mathrm{~m}$ & $1 \mathrm{~K}$ & Circling with $n$ Cycles (C) 1-Cycles & $1-10 \% 10-100 \%$ \\
\hline 5 & $100 \mathrm{~m}$ & $1 \mathrm{~K}, 5 \mathrm{~K}, 10 \mathrm{~K}$ & Mixed (M) & $1-10 \% 10-100 \%$ \\
\hline 6 & $100 \mathrm{~m}$ & $1 \mathrm{~K}, 5 \mathrm{~K}, 10 \mathrm{~K}$ & Mixed (M) & $1-10 \% 10-100 \%$ \\
\hline 7 & $100 \mathrm{~m}$ & $1 \mathrm{~K}$ & $\mathrm{O}, \mathrm{R} 1, \mathbf{R 2}, \mathrm{C}, \mathrm{M}$ & $1-10 \% 10-100 \%$ \\
\hline 8 & $100,500,1000 \mathrm{~m}$ & $1 \mathrm{~K}, 5 \mathrm{~K}$ & Mixed & $1-10 \% 10-100 \%$ \\
\hline 9 & $100,500,1000 \mathrm{~m}$ & $1 \mathrm{~K}, 5 \mathrm{~K}$ & Mixed & $1-10 \% 10-100 \%$ \\
\hline
\end{tabular}

\subsection{Results}

Table 1 summarises our parameter values for Melbourne city that we applied for our experimental settings. The selected map is an area of $10 \mathrm{~km} \times 10 \mathrm{~km}$. We selected $100 \mathrm{~m}$ grid cell size and $1 K$ trip numbers as our parameter values for the pre-study. We run 100 randomly selected QRs for each experiment to report an average for range query accuracy results. We compare all the baseline approaches with CASE in terms of accuracy in Section 8.4 with respect to query rectangle size, trip types, number of trips, and grid cell size as our experimental study parameters.

Table 2 Runtime Measurement of Histogram Construction for Melbourne City with respect to Grid Cell Size, and No. of Trips.

\begin{tabular}{|c|c|c|c|}
\hline & No. of Trips & Grid Cell Size & Histogram Construction Time \\
\hline \hline 1 & $1 \mathrm{~K}$ & $100 \mathrm{~m}$ & $9.665 \mathrm{~s}$ \\
\hline 2 & $1 \mathrm{~K}$ & $500 \mathrm{~m}$ & $2.505 \mathrm{~s}$ \\
\hline 3 & $1 \mathrm{~K}$ & $1000 \mathrm{~m}$ & $2.393 \mathrm{~s}$ \\
\hline \hline 4 & $5 \mathrm{~K}$ & $100 \mathrm{~m}$ & $40.99 \mathrm{~s}$ \\
\hline 5 & $5 \mathrm{~K}$ & $500 \mathrm{~m}$ & $8.879 \mathrm{~s}$ \\
\hline 6 & $5 \mathrm{~K}$ & $1000 \mathrm{~m}$ & $7.704 \mathrm{~s}$ \\
\hline \hline 7 & $10 \mathrm{~K}$ & $100 \mathrm{~m}$ & $63.376 \mathrm{~s}$ \\
\hline 8 & $10 \mathrm{~K}$ & $500 \mathrm{~m}$ & $15.019 \mathrm{~s}$ \\
\hline 9 & $10 \mathrm{~K}$ & $1000 \mathrm{~m}$ & $12.586 \mathrm{~s}$ \\
\hline
\end{tabular}




\subsubsection{Running Time}

Table 2 illustrates a summary of running time for the histogram construction related to the city of Melbourne with respect to various dataset and grid cell sizes. As shown, the larger grid cell size, the faster histograms will be constructed. The maximum time is related to $10 \mathrm{~K}$ trips and $100 \mathrm{~m}$ grid cell size, which is 63.376 seconds. As depicted, the pre-computational process is quite fast.

In regard to the range query, Table 3 demonstrates the measurement for the query response time of various dataset, grid cell, and QR sizes of Melbourne road network. As illustrated, given $10 \mathrm{~K}$ dataset size of Melbourne city, 100m grid cell size, and running a range query on the whole area, it will only take 36.8 seconds, which is still fast.

Table 3 Runtime Measurement of Range Query Response Time for Melbourne City with respect to Grid Cell Size, No. of Trips, and QR Size.

\begin{tabular}{|c|c|c|c|}
\hline No. of Trips & Grid Cell Size & QR Size & Query Response Time \\
\hline $1 \mathrm{~K}$ & $100 \mathrm{~m}$ & $1 \%$ & $0.001 \mathrm{~s}$ \\
\hline $1 \mathrm{~K}$ & $100 \mathrm{~m}$ & $5 \%$ & $0.005 \mathrm{~s}$ \\
\hline $1 \mathrm{~K}$ & $100 \mathrm{~m}$ & $10 \%$ & $0.032 \mathrm{~s}$ \\
\hline $1 \mathrm{~K}$ & $100 \mathrm{~m}$ & $50 \%$ & $0.247 \mathrm{~s}$ \\
\hline $1 \mathrm{~K}$ & $100 \mathrm{~m}$ & $100 \%$ & $0.448 \mathrm{~s}$ \\
\hline $1 \mathrm{~K}$ & $500 \mathrm{~m}$ & $1 \%$ & $0.001 \mathrm{~s}$ \\
\hline $1 \mathrm{~K}$ & $500 \mathrm{~m}$ & $10 \%$ & $0.003 \mathrm{~s}$ \\
\hline $1 \mathrm{~K}$ & $500 \mathrm{~m}$ & $100 \%$ & $0.074 \mathrm{~s}$ \\
\hline $1 \mathrm{~K}$ & $1000 \mathrm{~m}$ & $1 \%$ & $0.0 \mathrm{~s}$ \\
\hline $1 \mathrm{~K}$ & $1000 \mathrm{~m}$ & $10 \%$ & $0.002 \mathrm{~s}$ \\
\hline $1 \mathrm{~K}$ & $1000 \mathrm{~m}$ & $100 \%$ & $0.034 \mathrm{~s}$ \\
\hline $5 \mathrm{~K}$ & $100 \mathrm{~m}$ & $1 \%$ & $0.01 \mathrm{~s}$ \\
\hline $5 \mathrm{~K}$ & $100 \mathrm{~m}$ & $10 \%$ & $0.57 \mathrm{~s}$ \\
\hline $5 \mathrm{~K}$ & $100 \mathrm{~m}$ & $100 \%$ & $9.926 \mathrm{~s}$ \\
\hline $5 \mathrm{~K}$ & $500 \mathrm{~m}$ & $1 \%$ & $0.001 \mathrm{~s}$ \\
\hline $5 \mathrm{~K}$ & $500 \mathrm{~m}$ & $5 \%$ & $0.099 \mathrm{~s}$ \\
\hline $5 \mathrm{~K}$ & $500 \mathrm{~m}$ & $10 \%$ & $1.636 \mathrm{~s}$ \\
\hline$\overline{5 K}$ & $1000 \mathrm{~m}$ & $1 \%$ & $0.0 \mathrm{~s}$ \\
\hline $5 \mathrm{~K}$ & $1000 \mathrm{~m}$ & $10 \%$ & $0.005 \mathrm{~s}$ \\
\hline $5 \mathrm{~K}$ & $1000 \mathrm{~m}$ & $100 \%$ & $0.764 \mathrm{~s}$ \\
\hline $10 \mathrm{~K}$ & $100 \mathrm{~m}$ & $1 \%$ & $0.035 \mathrm{~s}$ \\
\hline $10 \mathrm{~K}$ & $100 \mathrm{~m}$ & $10 \%$ & $1.245 \mathrm{~s}$ \\
\hline $10 \mathrm{~K}$ & $100 \mathrm{~m}$ & $100 \%$ & $36.8 \mathrm{~s}$ \\
\hline $10 \mathrm{~K}$ & $500 \mathrm{~m}$ & $1 \%$ & $0.01 \mathrm{~s}$ \\
\hline $10 \mathrm{~K}$ & $500 \mathrm{~m}$ & $10 \%$ & $0.275 \mathrm{~s}$ \\
\hline $10 \mathrm{~K}$ & $500 \mathrm{~m}$ & $100 \%$ & $5.64 \mathrm{~s}$ \\
\hline $10 \mathrm{~K}$ & $1000 \mathrm{~m}$ & $1 \%$ & $0.005 \mathrm{~s}$ \\
\hline $10 \mathrm{~K}$ & $1000 \mathrm{~m}$ & $10 \%$ & $0.04 \mathrm{~s}$ \\
\hline $10 \mathrm{~K}$ & $1000 \mathrm{~m}$ & $100 \%$ & $2.62 \mathrm{~s}$ \\
\hline
\end{tabular}

\subsubsection{Query Rectangle Sizes and Trip Types}

Given a grid cell size of 100 meters, we run range queries for various sizes of the selected region, from $1 \%$ to $10 \%$ and $10 \%$ to $100 \%$, and for different types of trips. These correspond to Figure 21a-21h. Rows 1-4 of Table 1 show the parameter values for this part of the experiment. In the pre-study, we also experimented with larger numbers of trips with no significant difference identified ( $c f$. also Section 8.4.3 and Figure 23). We selected three 


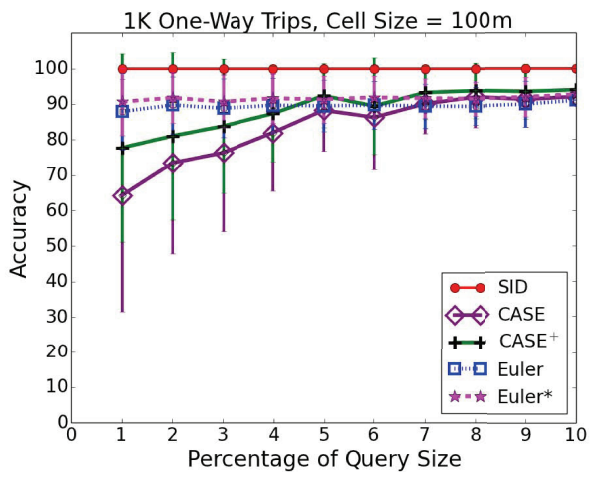

(a) One-way Trip, 1-10\% QR Size.

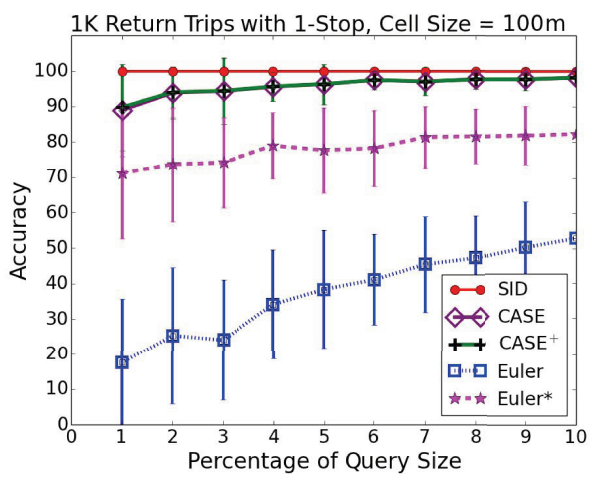

(c) Return Trip with 1-Stop, 1-10\% QR Size.

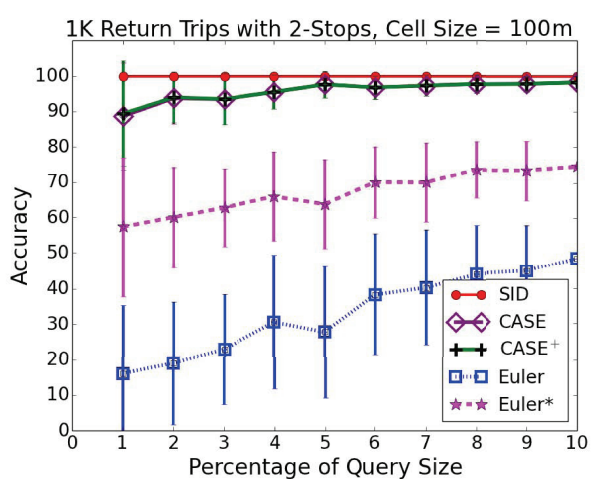

(e) Return Trip with 2-Stops, 1-10\% QR Size.

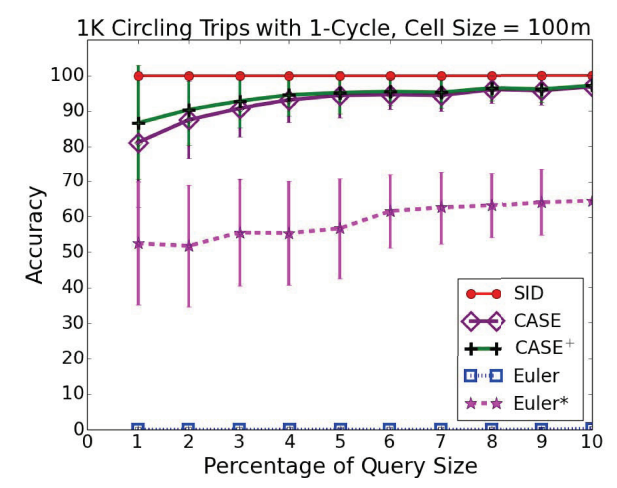

(g) Circling Trip with 1-Cycle, 1-10\% QR Size.

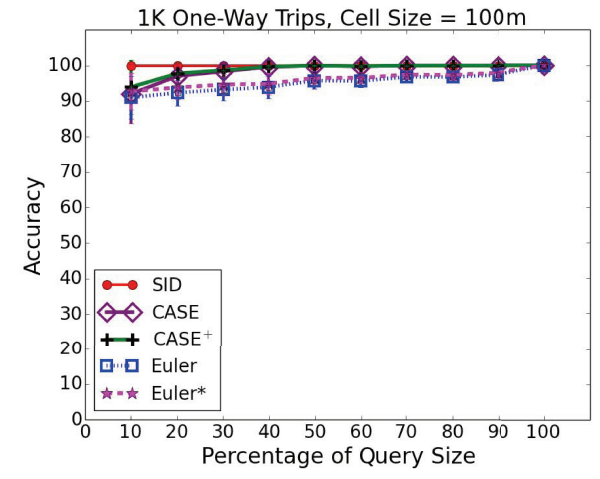

(b) One-way Trip, 10-100\% QR Size.

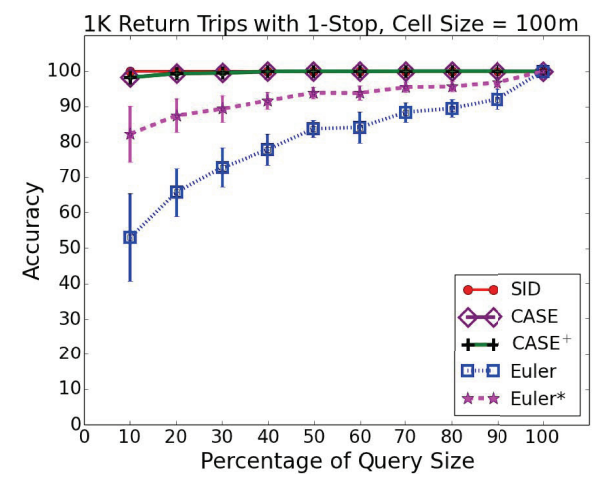

(d) Return Trip with 1-Stop, 10-100\% QR Size.

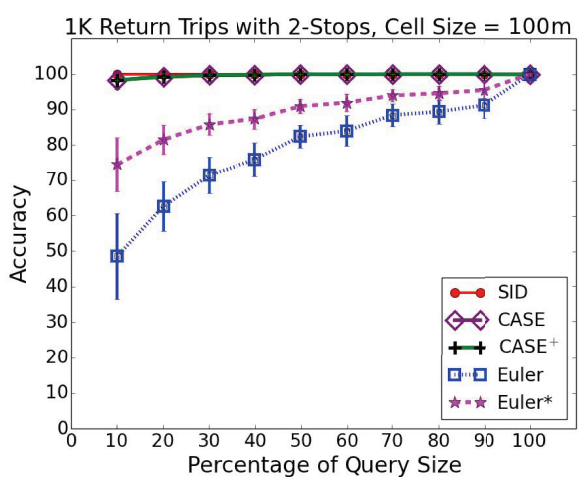

(f) Return Trip with 2-Stops, 10-100\% QR Size.

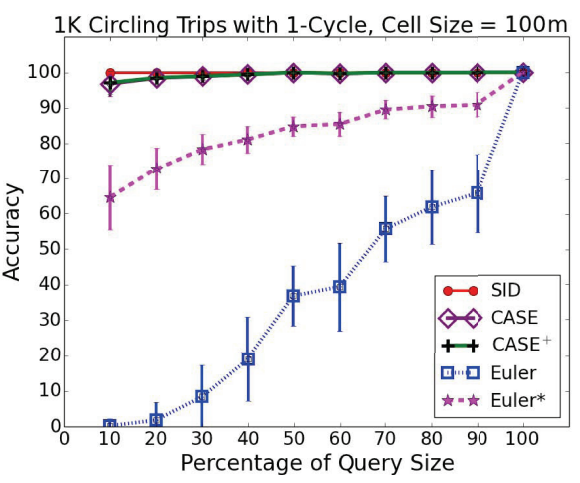

(h) Circling Trip with 1-Cycle, 10-100\% QR Size.

Fig. 21 Accuracy per Query Size in Melbourne, Trip Types. 
sizes for the trip numbers, $1 K, 10 K$ and $50 K$ as our pre-study evaluation. We selected the best competitive methods Euler and Euler ${ }^{\star}$ for our evaluation results. The only case where Euler and Euler ${ }^{\star}$ can outperform the CASE histogram is for QRs less than 5\% for one-way trips, Figure 21a, which is due to virtual counts, (cf. Examples10 and 11 in Section 5). This implies that since one-way trips are created randomly, it may happen that the shape of a trip has a returning shape, which leads to creating virtual counts and inaccuracy in small QRs. Euler ${ }^{\star}$ is expected to provide answers with a greater level of accuracy than Euler. Euler indeed has lower accuracy than Euler ${ }^{\star}$, since Euler ${ }^{\star}$ leverages additional information, i.e., the full trajectory in the pre-processing phase. As depicted in Figure 21, overall, CASE provides a higher level of accuracy whereas $\mathrm{CASE}^{+}$outperforms CASE for smaller QR.

As discussed in Section 8.2, we have considered different trip patterns to make simulate a more realistic scenario. In Figure 21, the accuracy level of the various trip types is depicted. Except for the one-way trip and QRs smaller than 5\% (discussed in the previous paragraph), CASE outperforms other techniques for both small and large QRs across all experiments. As expected and shown in Figure 21, for those types of trips that cause the distinct counting problem, return trips, circling trips with $n$ cycles and mixed trips (cf. Section8.2), CASE significantly outperforms Euler and Euler ${ }^{\star}$. This implies that the concept of using virtual lines improves the accuracy level in range query close to $100 \%$ for smaller QRs; for larger QRs we have $100 \%$ accuracy. In order to examine the effect of having one or more cycles in trips, we have studied this parameter further ( $c f$. Figure $21 \mathrm{~g}$ and $21 \mathrm{~h}$ ). The number of cycles in this figure is 1 and the results are shown for different techniques. As depicted, Euler suffers low accuracy. Euler ${ }^{\star}$ does not change considerably in comparison with return trips, however, the accuracy for the $1-10 \%$ query sizes is lower than CASE, $\approx 50 \%$ accuracy. This is exactly related to our discussion about having a cycle or returning path in a trip which leads to the distinct counting problem ( $c f$. Section 8.2.3). Figure 22, shows the accuracy per

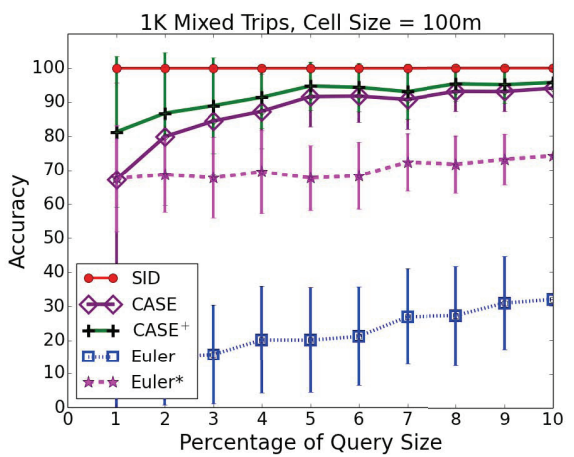

(a) $1-10 \%$ QR Size.

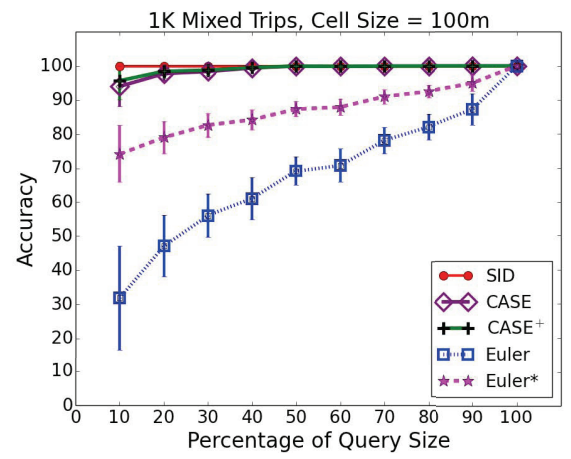

(b) $10-100 \%$ QR Size.

Fig. 22 Accuracy per Query Size for 1K Mixed Trips in Melbourne, Small and Large QRs.

query size for $1 \mathrm{~K}$ mixed trips. As explained in Section 8.2, a mixed trip is a combination of one-way, return (with 1-stop and 2-stops) and circling trips with 1 cycle. This trip is a realistic trip for every day trip patterns. Figure 22 demonstrates that CASE outperforms other techniques even if in $25 \%$ of the trips (one-way trip) virtual counts lead to inaccuracy for the smaller QRs. 


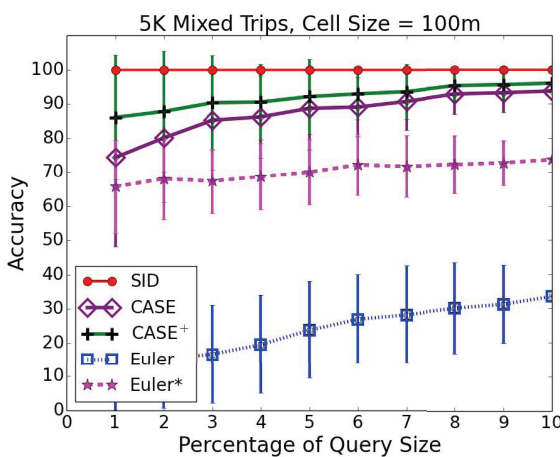

(a) 5K Mixed Trips, 1-10\% QR Size.

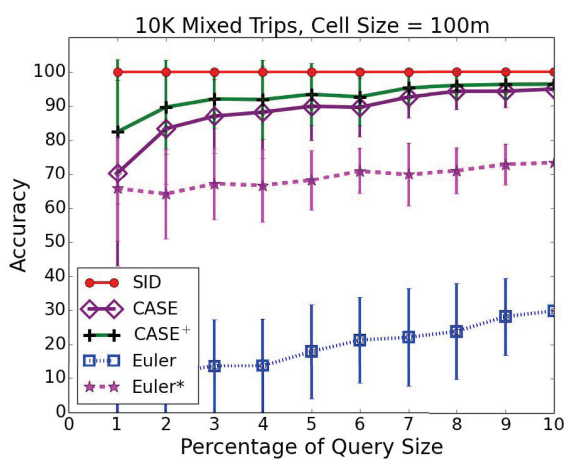

(c) 10K Mixed Trips, 1-10\% QR Size.

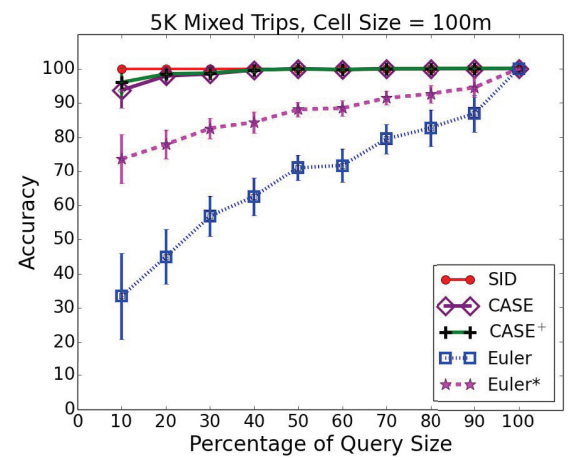

(b) 5K Mixed Trips, 10-100\% QR Size.

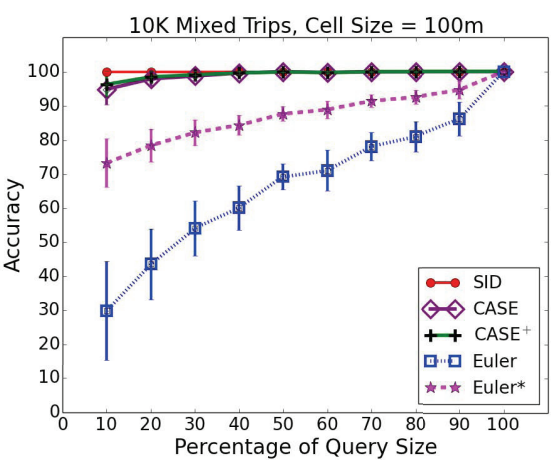

(d) 10K Mixed Trips, 10-100\% QR Size.

Fig. 23 Accuracy per Query Size, Large Trip Numbers in Melbourne, Small and Large QRs.

We experiment with varying numbers of trips which shows that the pattern in larger number of trips does not change overall. Figures $23 \mathrm{a}$ and $23 \mathrm{~b}$ show the results for $5 \mathrm{k}$ mixed trips for small and large query rectangle sizes. We also experimented with $10 \mathrm{~K}$ mixed trips of small and large query rectangle sizes (Figures 23c and 23d). For the larger query rectangle sizes, there is not a considerable change in the pattern. However, as depicted in Figures 23a and $23 \mathrm{c}, \mathrm{CASE}^{+}$has a slightly higher level of accuracy in smaller QRs for larger number of trips in comparison with CASE and Euler ${ }^{\star}\left(c f\right.$. Figure 22a). This advantage of $\mathrm{CASE}^{+}$, for smaller QRs, is explored in detail in Section 8.7.

In order to have a realistic evaluation we selected mixed trips as default trip type to run the rest of the experiments ( $c f$. Section 8.2.4).

\subsubsection{Grid Cell Sizes}

In this part of the experiment we study the result of changing the granularity of the grid. We have considered various sizes of the grid cell, 100, 500, and 1000m for Melbourne 
city. In Figure 24 and 25 the accuracy results for $1 K$ and $5 K$ mixed trips and two larger cell sizes, 500, and $1000 \mathrm{~m}$ are shown. Considering the variety of the grid cells, the CASE histogram is independent of the changes to the grid cell size and always provides a high level of accuracy. However, other techniques perform better in larger grid cell sizes. The only setting that CASE and $\mathrm{CASE}^{+}$have an accuracy of $100 \%$ or $\approx 100 \%$ for smaller QRs, is when the grid cell size is aligned with $1 \%$ QR size, ( $c f$. Figures $24 \mathrm{c}$ and $25 \mathrm{c}$ ).

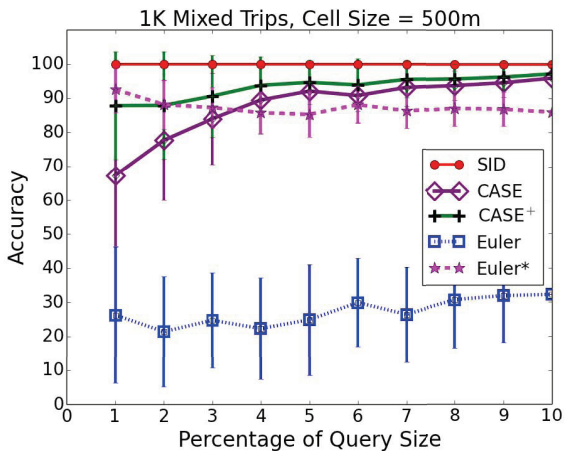

(a) $1-10 \%$ QR Size.

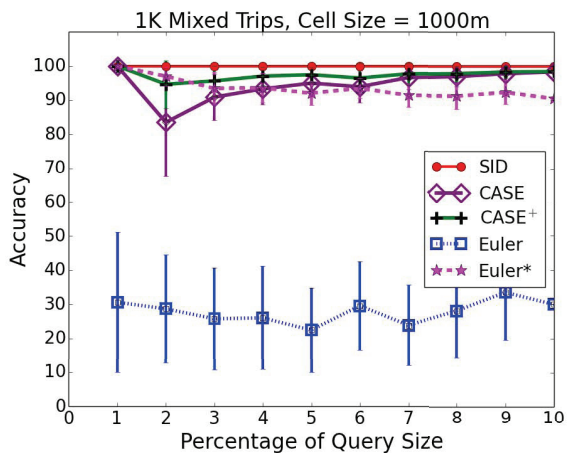

(c) $1-10 \%$ QR Size.

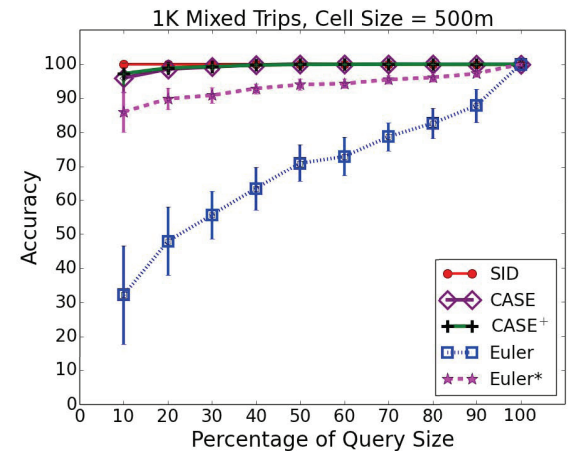

(b) $10-100 \%$ QR Size.

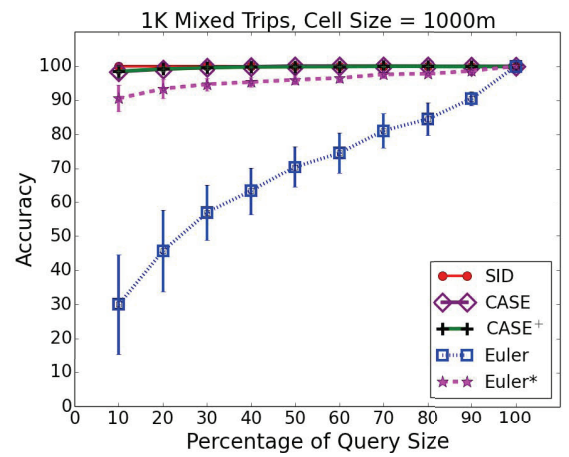

(d) $10-100 \%$ QR Size.

Fig. 24 Accuracy per Query Size, Various Grid Cell Sizes in Melbourne for 1K Trips.

All experiments show the same trend for the other techniques, Euler and Euler ${ }^{\star}$, that they cannot achieve a high level of accuracy until we increase the size of the grid cell. When it is equal to an extreme size of $1000 m$, i.e., $1 \%$ of the deployment area, Euler ${ }^{\star}$ significantly improves. Euler and Euler ${ }^{\star}$ histograms are increasing with the query rectangle size. The reason for this observation is that the smaller grid cell size increases the probability of the distinct counting problem as each moving object passes more partitions. We show various cases for the QR size in Figures 24a, 25c, 25a, and 25c (see also Figures 22 and 23 that use the $100 \mathrm{~m}$ grid cell size).

All these experiments show that the CASE histogram achieves near perfect accuracy except for very small query rectangle size ranging between 1-5\%. CASE still typically achieves 


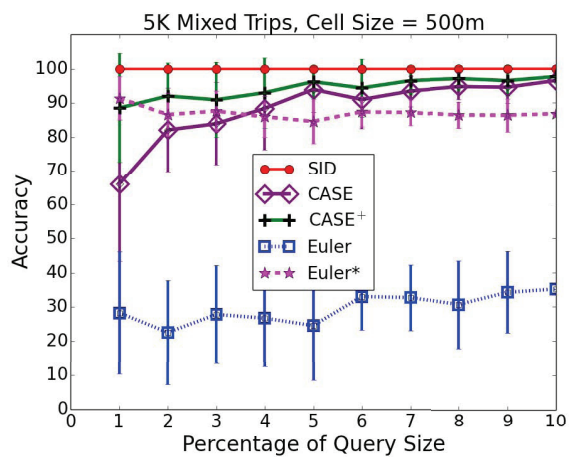

(a) $1-10 \%$ QR Size.

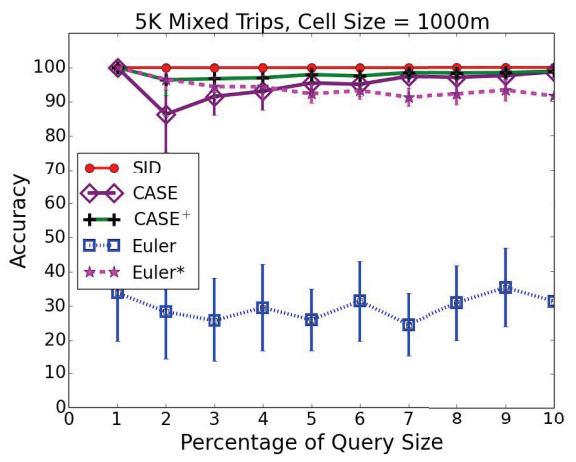

(c) $1-10 \%$ QR Size.

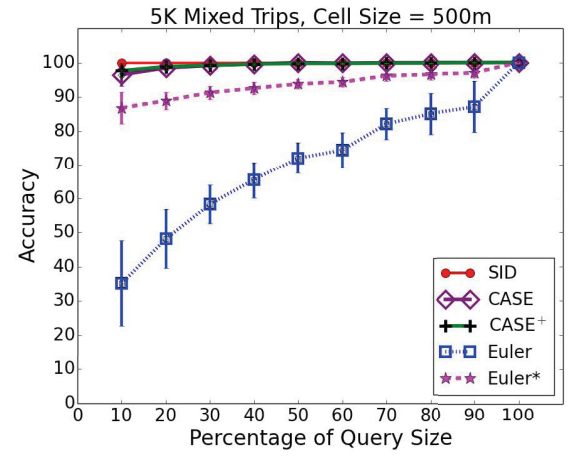

(b) $10-100 \%$ QR Size.

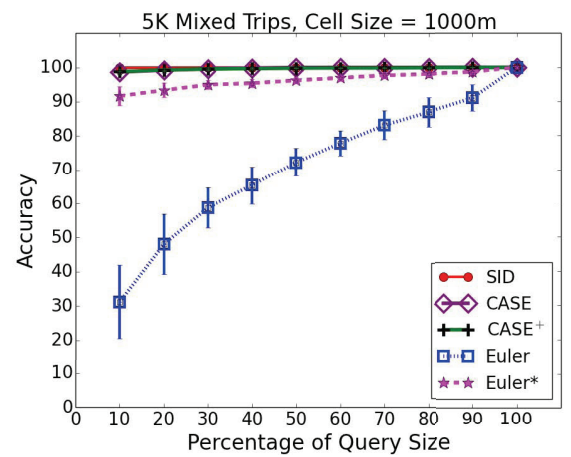

(d) $10-100 \%$ QR Size.

Fig. 25 Accuracy per Query Size, Various Grid Cell Sizes in Melbourne for 5K Trips.

a high accuracy in excess of $90 \%$ for the rest of the QR sizes and clearly outperforms comparable methods.

\subsection{Manhattan}

Table 4 summarises our selected parameter values for Manhattan. Figure 26 illustrates accuracy percentages for three different grid cell sizes, 100, 150 and $200 \mathrm{~m}$ per query size for small and large QRs in Manhattan for 5K mixed trips.

Table 4 Experimental settings for Manhattan. The bold items in a row are varied with the non-bold items are held fixed.

\begin{tabular}{|c|c|c|c|c|}
\hline & Grid Cell Size & No. of Trips & Trip Types & QR Size \\
\hline \hline 1 & $100 \mathrm{~m}$ & $5 \mathrm{~K}$ & Mixed & $\mathbf{1 - 1 0 \%} \mathbf{1 0 - 1 0 0 \%}$ \\
\hline 2 & $150 \mathrm{~m}$ & $5 \mathrm{~K}$ & Mixed & $\mathbf{1 - 1 0 \%} \mathbf{1 0 - 1 0 0 \%}$ \\
\hline 3 & $200 \mathrm{~m}$ & $5 \mathrm{~K}$ & Mixed & $\mathbf{1 - 1 0 \%} \mathbf{1 0 - 1 0 0 \%}$ \\
\hline
\end{tabular}




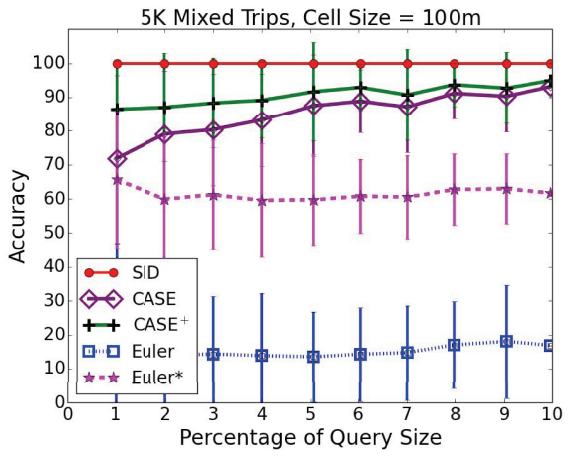

(a) $1-10 \%$ QR Size, Cell Size 100m.

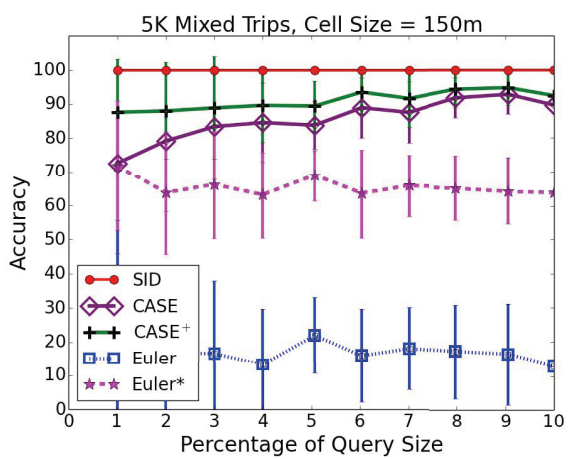

(c) $1-10 \%$ QR Size, Cell Size 150m.

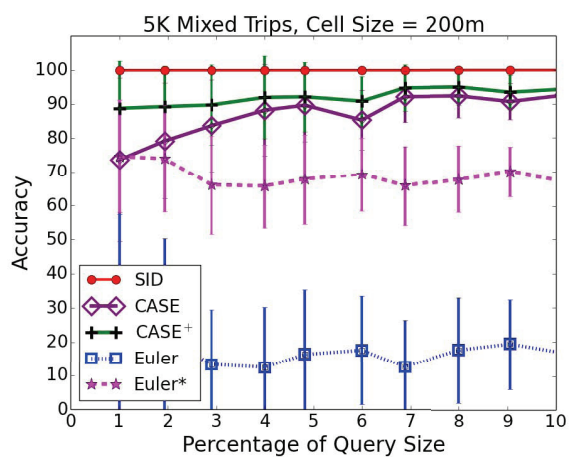

(e) $1-10 \%$ QR Size, Cell Size 200m.

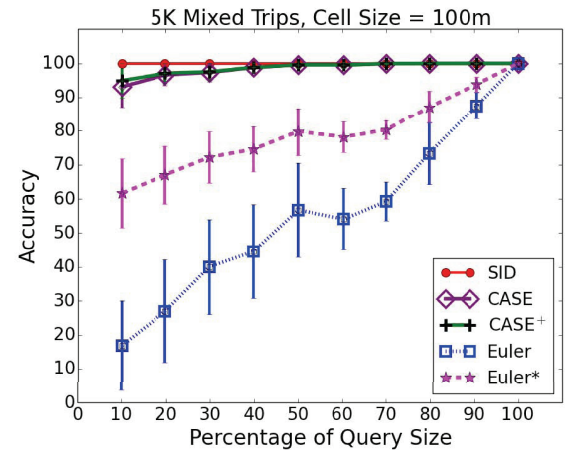

(b) $10-100 \%$ QR Size, Cell Size 100m.

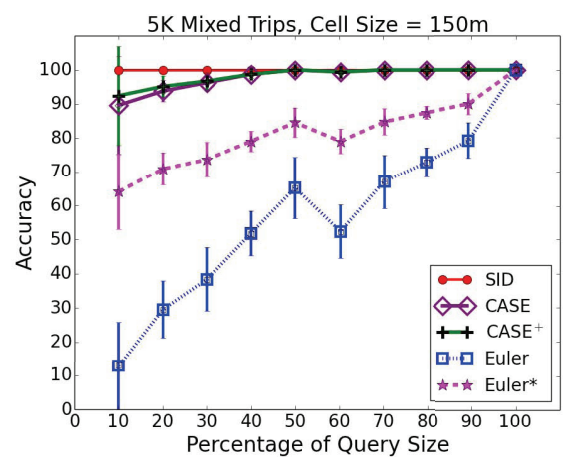

(d) $10-100 \%$ QR Size, Cell Size 150m.

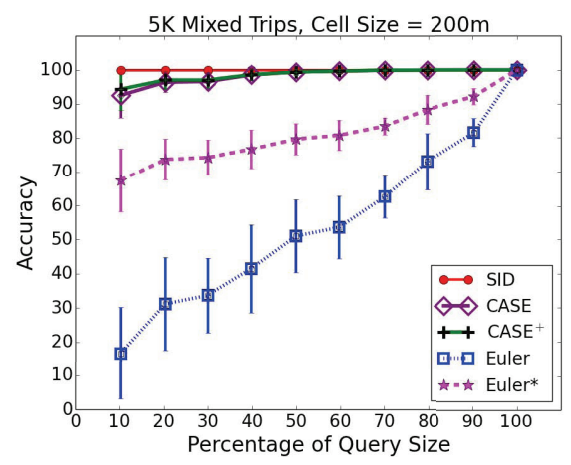

(f) $10-100 \%$ QR Size, Cell Size 200m.

Fig. 26 Accuracy per Query Size for 5K Mixed Trips in Manhattan, Small and Large QRs.

Figure 26 depicts similar accuracy results for a different spatial layer, on the Manhattan road network, for CASE and $\mathrm{CASE}^{+}$. Regarding the different grid cell sizes, as expected by increasing the grid cell size from $100 \mathrm{~m}, 150$ to $200 \mathrm{~m}$, Euler ${ }^{\star}$ performs better, (see 
Figures 26a, 26c and 26e). However, the accuracy level does not change for our proposed approach.

\subsection{Beijing}

Figures 27 and 28 illustrate accuracy percentages per query size for small and large QRs in Beijing for mixed and circling trips. The circling trips have also been selected for our comparison on the real dataset. Table 5 summarises our selected parameter values for this city. As illustrated in Figure 27, we see similar results to our previous road networks. Figure 28 shows that having a cycle in a trip reduces the accuracy for Euler ${ }^{\star}$ and Euler. The accuracy will be dramatically reduced when we have different shapes of circling, returning, self-intersecting in a trip, (see Figure 30), which is the case in our real dataset to be discussed in the next section.

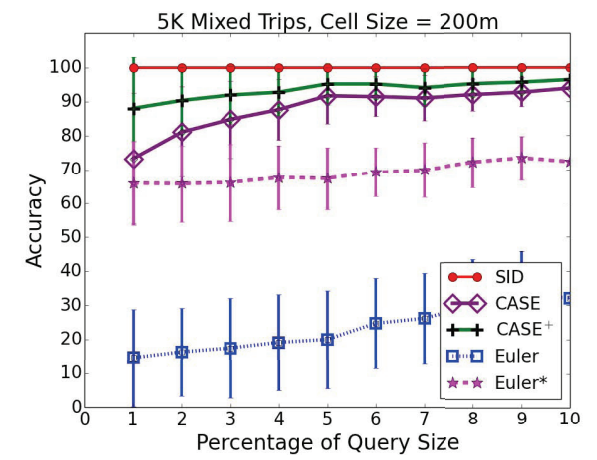

(a) $1-10 \%$ QR Size.

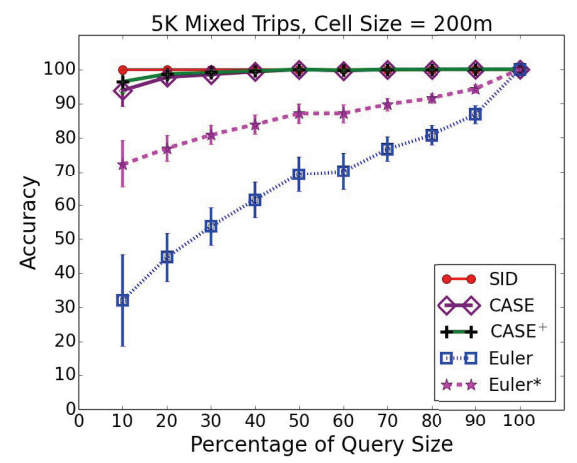

(b) $10-100 \%$ QR Size.

Fig. 27 Accuracy per Query Size for 5K Mixed Trips in Beijing, Small and Large QRs.

Table 5 Experimental settings for Beijing. Bold items in a row are varied, while non-bold items are held fixed.

\begin{tabular}{|c|c|c|c|c|}
\hline & Grid Cell Size & No. of Trips & Trip Types & QR Size \\
\hline \hline 1 & $200 \mathrm{~m}$ & $5 \mathrm{~K}$ & Mixed & $\mathbf{1 - 1 0 \% ~ 1 0 - 1 0 0 \%}$ \\
\hline 2 & $200 \mathrm{~m}$ & $1 \mathrm{~K}$ & Circling with n Cycles (C) 1-Cycles & $\mathbf{1 - 1 0 \% ~ 1 0 - 1 0 0 \%}$ \\
\hline
\end{tabular}

\subsubsection{Real-World Dataset: T-Drive}

We also compare our algorithm with other competitive methods on the T-Drive dataset which is publicly available on Microsoft's website [52,53]. Since the dataset refers to taxi drivers, the trajectories are mostly circling trips with a large number of self-intersecting patterns. This dataset confirms the importance of return and circling trips investigated in Section 8.2. The area size is $20 \mathrm{~km} * 20 \mathrm{~km}$ and we consider the same grid cell size of $200 \mathrm{~m}$ for synthetic 


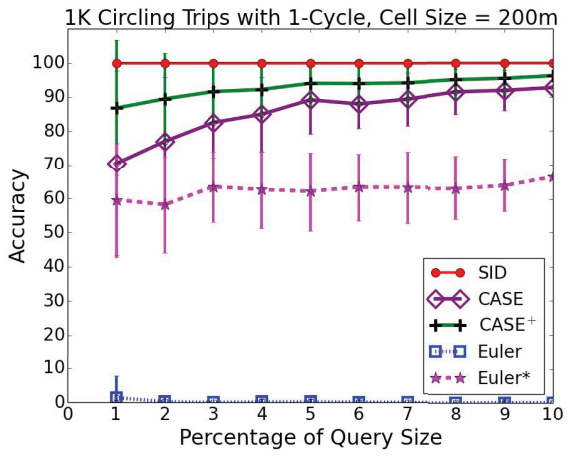

(a) $1-10 \%$ QR Size.

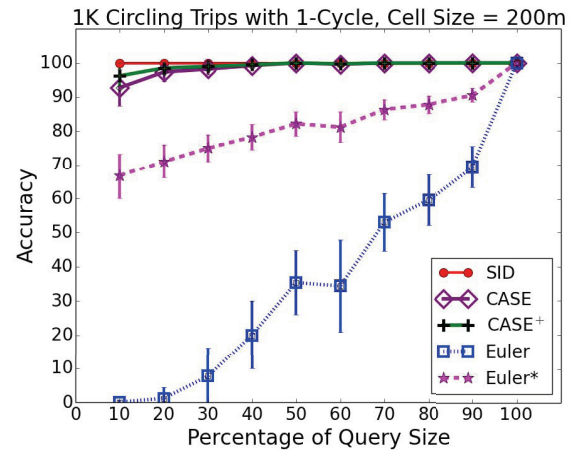

(b) $10-100 \%$ QR Size.

Fig. 28 Accuracy per Query Size for 5K Circling Trips in Beijing, Small and Large QRs.

Beijing trajectories, to determine differences in terms of accuracy. Figure 29 demonstrates the results for the selected grid cell size $200 \mathrm{~m}$, with CASE outperforming Euler approaches since it considers vertex counts. Regarding the smaller QRs, especially for a QR that is $1 \%$ of the total area, $\mathrm{CASE}^{+}$is more accurate than CASE, because it removes some unnecessary virtual counts. Both Euler and Euler ${ }^{\star}$ techniques are significantly more inaccurate because of the trajectories' shape. Our technique CASE achieves high accuracy exceeding $90 \%$ once the QR is greater than $5 \%$.

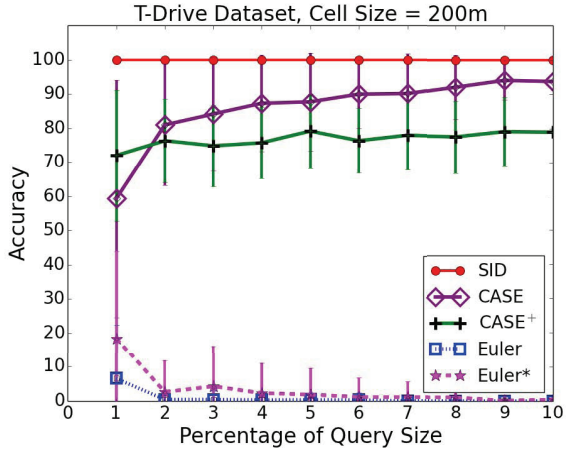

(a) $1-10 \%$ QR Size.

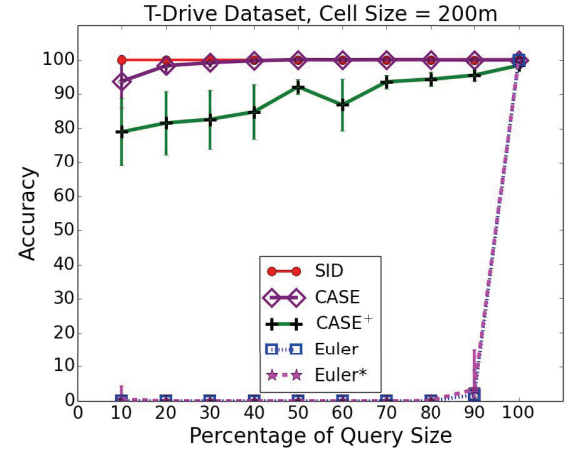

(b) $10-100 \%$ QR Size.

Fig. 29 Accuracy per Query Size for T-Drive Dataset of Beijing City. A Real Example of Trajectory Trip Patterns.

Figure 30 illustrates two sample trajectories from the T-Drive dataset, which shows their trip shapes. As depicted, this dataset consists of various shapes of trajectories, e.g., returning, circling and self-intersecting trips. As a result, applying virtual counts in the CASE method to improve the accuracy of the results are confirmed, and $\mathrm{CASE}^{+}$can merely outperform CASE for a very small QR like $1 \%$. 


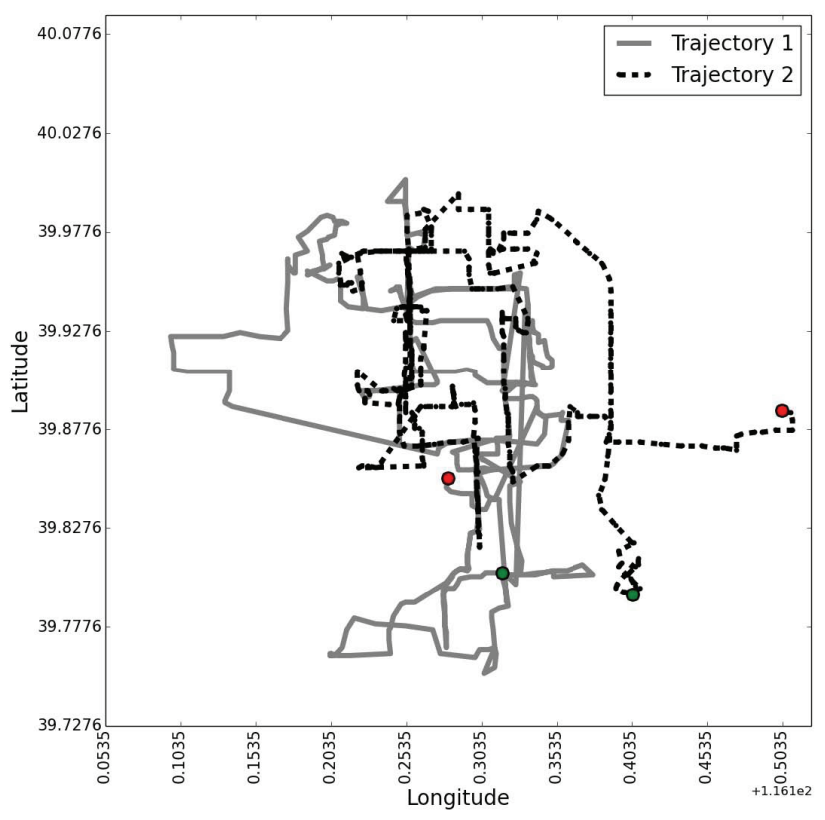

Fig. 30 Sample trajectory visualization for T-Drive Dataset.

\subsection{CASE versus $\mathrm{CASE}^{+}$}

CASE and $\mathrm{CASE}^{+}$both perform well overall. $\mathrm{CASE}^{+}$can outperform CASE for the smaller QRs whereas CASE is better when the ratio of the grid cell size of the monitored area is large and QR is large as well. We run a suite of experiments to confirm this. All the experiments demonstrate the $\mathrm{CASE}^{+}$evaluation, for smaller QR sizes.

We study $\mathrm{CASE}^{+}$for the smaller QRs to demonstrate accuracy in Sections 8.4.2 and 8.4.4, which shows the result for the $\mathrm{CASE}^{+}$algorithm which aims to improve accuracy level. As discussed earlier, the error in the CASE approach occurs when we have a smaller QR. Therefore we run the experiment for the smaller QR starting from $1 \%$ of the QR to see the difference between CASE and $\mathrm{CASE}^{+}$in the result. As shown in Figure 21, $\mathrm{CASE}^{+}$ could achieve a better accuracy level than CASE. CASE and $\mathrm{CASE}^{+}$can complement each other for various settings. As depicted in Figure 29, CASE has a better accuracy when the $\mathrm{QR}$ is larger or for coarse grid cell sizes.

\section{Conclusion}

In this paper, we propose CASE as a connectivity aware spatial Euler histogram to solve the distinct-object counting problem. In CASE histograms, the connectivity of trajectory paths is preserved while privacy is maintained through the use of count information. The key idea of CASE is to use virtual counts to address the distinct counting problem. Our technique outperforms the best known methods in terms of maintaining a high-level of accuracy in the provided answers. We provide a new analysis of Euler histogram based approaches 
demonstrating worst-case privacy, and general lower bounds on privacy. We also contribute a wrapper mechanism that operates with Euler histogram approaches to answer interactive range queries with preserving the strong notion of differential privacy. In the experimental study, we test our approach over a range of realistic scenarios by considering different types of moving object patterns as well as using a real trajectory dataset, T-Drive. The results show that CASE significantly outperforms other approaches. Our results demonstrate that CASE histograms can always achieve a high level of accuracy and are accurate for larger query regions whereas Euler histograms suffer lower accuracy.

An important feature of CASE is that its performance is independent of the cell size. This observation implies that whatever the grid cell size the application designer requires, CASE will always provide a high level of accuracy. Although in this paper we focused on trajectory data, our method is applicable to other type of objects, e.g., polygons. We have identified the source of error in the CASE approach for smaller query sizes and have initiated an approach to improving accuracy towards $100 \%$ with $\mathrm{CASE}^{+}$.

Interesting questions for future work coming out of this paper, include: deriving further bounds on confusion level given histogram counts, interactive approaches to differential privacy for Euler histograms, deriving bounds on accuracy under some data assumptions, and performing experimental evaluation of the effect of query region and partition geometries on accuracy.

Acknowledgements We thank Dr. Hairuo Xie for his helpful comments regarding the experimental study.

\section{References}

1. Auguste kerckhoffs. Journal des sciences militaires IX, 5-38 (1883)

2. Barak, B., Chaudhuri, K., Dwork, C., Kale, S., McSherry, F., Talwar, K.: Privacy, accuracy, and consistency too: a holistic solution to contingency table release. In: Proceedings of the Twenty-Sixth ACM SIGACT-SIGMOD-SIGART Symposium on Principles of Database Systems, June 11-13, 2007, Beijing, China, pp. 273-282 (2007)

3. Beigel, R., Tanin, E.: The geometry of browsing. In: LATIN '98: Theoretical Informatics, Third Latin American Symposium, pp. 331-340 (1998)

4. Beresford, A.R., Stajano, F.: Location privacy in pervasive computing. IEEE Pervasive Computing 2(1), 46-55 (2003)

5. Beresford, A.R., Stajano, F.: Mix zones: User privacy in location-aware services. In: 2nd IEEE Conference on Pervasive Computing and Communications Workshops (PerCom 2004 Workshops), pp. 127-131 (2004)

6. Bogorny, V., Shekhar, S.: Spatial and spatio-temporal data mining. In: ICDM 2010, The 10th IEEE International Conference on Data Mining, p. 1217 (2010)

7. Braz, F., Orlando, S., Orsini, R., Raffaetà, A., Roncato, A., Silvestri, C.: Approximate aggregations in trajectory data warehouses. In: Proceedings of the 23rd International Conference on Data Engineering Workshops, ICDE 2007, pp. 536-545 (2007)

8. Buchin, K., Buchin, M., van Kreveld, M.J., Löffler, M., Luo, J., Silveira, R.I.: Processing aggregated data: the location of clusters in health data. GeoInformatica 16(3), 497-521 (2012)

9. Chawla, S., Dwork, C., McSherry, F., Talwar, K.: On the utility of privacy-preserving histograms. In: Proceedings of the 21st Conference on Uncertainty in Artificial Intelligence (2005)

10. Chow, C.Y., Mokbel, M.F.: Privacy of spatial trajectories. In: Computing with Spatial Trajectories, pp. 109-141 (2011)

11. Chow, C.Y., Mokbel, M.F.: Trajectory privacy in location-based services and data publication. SIGKDD Explorations 13(1), 19-29 (2011)

12. Dingledine, R., Mathewson, N., Syverson, P.F.: Tor: The second-generation onion router. In: Proceedings of the 13th USENIX Security Symposium, August 9-13, 2004, San Diego, CA, USA, pp. 303-320 (2004)

13. Dwork, C.: Differential privacy: A survey of results. In: Theory and Applications of Models of Computation, 5th International Conference, TAMC 2008, Xi'an, China, April 25-29, 2008. Proceedings, pp. $1-19(2008)$ 
14. Dwork, C., McSherry, F., Nissim, K., Smith, A.: Calibrating noise to sensitivity in private data analysis. In: Theory of Cryptography, Third Theory of Cryptography Conference, TCC 2006, New York, NY, USA, March 4-7, 2006, Proceedings, Lecture Notes in Computer Science, vol. 3876, pp. 265-284. Springer (2006)

15. Dwork, C., Naor, M., Pitassi, T., Rothblum, G.N., Yekhanin, S.: Pan-private streaming algorithms. In: Innovations in Computer Science - ICS 2010, Tsinghua University, Beijing, China, January 5-7, 2010. Proceedings, pp. 66-80 (2010)

16. Giannotti, F., Nanni, M., Pinelli, F., Pedreschi, D.: Trajectory pattern mining. In: Proceedings of the 13th ACM SIGKDD International Conference on Knowledge Discovery and Data Mining, pp. 330-339 (2007)

17. Gómez, L.I., Kuijpers, B., Moelans, B., Vaisman, A.A.: A state-of-the-art in spatio-temporal data warehousing, OLAP and mining. In: Integrations of Data Warehousing, Data Mining and Database Technologies, pp. 200-236 (2011)

18. Gruteser, M., Liu, X.: Protecting privacy in continuous location-tracking applications. IEEE Security \& Privacy 2(2), 28-34 (2004)

19. Hai, Zhang, R., Zheng, Y., Xie, X., Huang, J., Xu, Z.: Destination prediction by sub-trajectory synthesis and privacy protection against such prediction. In: 29th IEEE International Conference on Data Engineering, ICDE 2013, pp. 254-265 (2013)

20. Jeung, H., Yiu, M.L., Jensen, C.S.: Trajectory pattern mining. In: Computing with Spatial Trajectories, pp. 143-177 (2011)

21. Krumm, J.: Inference attacks on location tracks. In: Pervasive Computing, 5th International Conference, PERVASIVE 2007, pp. 127-143 (2007)

22. Leonardi, L., Orlando, S., Raffaetà, A., Roncato, A., Silvestri, C., Andrienko, G.L., Andrienko, N.V.: A general framework for trajectory data warehousing and visual OLAP. GeoInformatica 18(2), 273-312 (2014)

23. Loo, B.P.: Validating crash locations for quantitative spatial analysis: A GIS-based approach. Accident Analysis \& Prevention 38(5), 879-886 (2006)

24. López, I.F.V., Snodgrass, R.T., Moon, B.: Spatiotemporal aggregate computation: a survey. IEEE Transactions on Knowledge and Data Engineering, TKDE 17(2), 271-286 (2005)

25. Marketos, G., Frentzos, E., Ntoutsi, I., Pelekis, N., Raffaetà, A., Theodoridis, Y.: Building real-world trajectory warehouses. In: Seventh ACM International Workshop on Data Engineering for Wireless and Mobile Access, Mobide 2008, pp. 8-15 (2008)

26. MicrosoftNewsCenter: Data privacy day tackles concerns as location-based services grow in popularity. http://www.microsoft.com/en-us/news/features/2011/jan11/01-26dataprivacyday.aspx (2011). Accessed: 2013-09-15

27. Narayanan, A.: Data privacy: The non-interactive setting. Ph.D. thesis, Austin, TX, USA (2009). AAI3368859

28. OpenStreetMap: The free wiki world map. http://www.openstreetmap.org/. Accessed: 2013-05-10

29. Orlando, S., Orsini, R., Raffaetà, A., Roncato, A., Silvestri, C.: Spatio-temporal aggregations in trajectory data warehouses. In: Data Warehousing and Knowledge Discovery, 9th International Conference, DaWaK 2007, pp. 66-77 (2007)

30. Orlando, S., Orsini, R., Raffaetà, A., Roncato, A., Silvestri, C.: Trajectory data warehouses: Design and implementation issues. Journal of Computing Science and Engineering, JCSE 1(2), 211-232 (2007)

31. Papadias, D., Kalnis, P., Zhang, J., Tao, Y.: Efficient OLAP operations in spatial data warehouses. In: Advances in Spatial and Temporal Databases, 7th International Symposium, SSTD 2001, pp. 443-459 (2001)

32. Pedersen, T.B., Tryfona, N.: Pre-aggregation in spatial data warehouses. In: Advances in Spatial and Temporal Databases, 7th International Symposium, SSTD 2001, pp. 460-480 (2001)

33. Phillips, P., Lee, I.: Crime analysis through spatial areal aggregated density patterns. GeoInformatica 15(1), 49-74 (2011)

34. Sakr, M.A., Güting, R.H.: Spatiotemporal pattern queries. GeoInformatica 15(3), 497-540 (2011)

35. Samet, H.: Foundations of multidimensional and metric data structures. Morgan Kaufmann (2006)

36. Sun, C., Agrawal, D., El Abbadi, A.: Exploring spatial datasets with histograms. In: Proceedings of the 18th International Conference on Data Engineering, ICDE, pp. 93-102 (2002)

37. Sun, C., Bandi, N., Agrawal, D., El Abbadi, A.: Exploring spatial datasets with histograms. Distributed and Parallel Databases 20(1), 57-88 (2006)

38. Sweeney, L.: k-anonymity: A model for protecting privacy. International Journal of Uncertainty, Fuzziness and Knowledge-Based Systems 10(5), 557-570 (2002)

39. Tao, Y., Kollios, G., Considine, J., Li, F., Papadias, D.: Spatio-temporal aggregation using sketches. In: Proceedings of the 20th International Conference on Data Engineering, ICDE 2004, pp. 214-225 (2004) 
40. Tao, Y., Papadias, D., Zhang, J.: Aggregate processing of planar points. In: Advances in Database Technology - EDBT 2002, 8th International Conference on Extending Database Technology, pp. 682700 (2002)

41. Timko, I., Böhlen, M.H., Gamper, J.: Sequenced spatio-temporal aggregation in road networks. In: EDBT 2009, 12th International Conference on Extending Database Technology, pp. 48-59 (2009)

42. Trudeau, R.: Introduction to Graph Theory. Dover Books on Mathematics Series. Dover Pub. (1993)

43. Viswanathan, G., Schneider, M.: On the requirements for user-centric spatial data warehousing and SOLAP. In: Database Systems for Adanced Applications - 16th International Conference, DASFAA 2011, International Workshops, pp. 144-155 (2011)

44. Wernke, M., Skvortsov, P., Dürr, F., Rothermel, K.: A classification of location privacy attacks and approaches. Personal and Ubiquitous Computing 18(1), 163-175 (2014)

45. Willer, D.J.: A spatial decision support system for bank location: A case study. Tech. rep., University of New York at Buffalo, Department of Geography State, National Center for Geographic Information and Analysis (1990)

46. Xie, H., Kulik, L., Tanin, E.: Privacy-aware traffic monitoring. IEEE Transactions on Intelligent Transportation Systems 11(1), 61-70 (2010)

47. Xie, H., Tanin, E., Kulik, L.: Distributed histograms for processing aggregate data from moving objects. In: 8th International Conference on Mobile Data Management (MDM 2007), pp. 152-157 (2007)

48. Xie, H., Tanin, E., Kulik, L., Scheuermann, P., Trajcevski, G., Fanaeepour, M.: Euler histogram tree: A spatial data structure for aggregate range queries on vehicle trajectories. In: 7th ACM SIGSPATIAL International Workshop on Computational Transportation Science, IWCTS 2014 (2014)

49. Xue, A.Y., Qi, J., Xie, X., Zhang, R., Huang, J., Li, Y.: Solving the data sparsity problem in destination prediction. The International Journal on Very Large Data Bases, VLDB J. (2014)

50. Xue, A.Y., Zhang, R., Zheng, Y., Xie, X., Yu, J., Tang, Y.: Desteller: A system for destination prediction based on trajectories with privacy protection. The Proceedings of the VLDB Endowment (PVLDB) 6(12), 1198-1201 (2013)

51. Yaagoub, A., Liu, X., Trajcevski, G., Tanin, E., Scheuermann, P.: Materialized views for count aggregates of spatial data. In: Advances in Databases and Information Systems - 16th East European Conference, ADBIS 2012, pp. 427-440 (2012)

52. Yuan, J., Zheng, Y., Xie, X., Sun, G.: Driving with knowledge from the physical world. In: Proceedings of the 17th ACM SIGKDD International Conference on Knowledge Discovery and Data Mining, pp. 316-324 (2011)

53. Yuan, J., Zheng, Y., Zhang, C., Xie, W., Xie, X., Sun, G., Huang, Y.: T-drive: driving directions based on taxi trajectories. In: 18th ACM SIGSPATIAL International Symposium on Advances in Geographic Information Systems, ACM-GIS 2010, pp. 99-108 (2010) 